Noname manuscript No.

(will be inserted by the editor)

\title{
Corners and stable optimized domain decomposition methods for the Helmholtz problem
}

\author{
B. Després, A. Nicolopoulos, B. Thierry
}

Received: date / Accepted: date

\begin{abstract}
We construct a new Absorbing Boundary Condition (ABC) adapted to solving the Helmholtz equation in polygonal domains in dimension two. Quasi-continuity relations are obtained at the corners of the polygonal boundary. This ABC is then used in the context of domain decomposition where various stable algorithms are constructed and analysed. Next, the operator of this $\mathrm{ABC}$ is adapted to obtain a transmission operator for the Domain Decomposition Method (DDM) that is well suited for broken line interfaces. For each algorithm, we show the decrease of an adapted quadratic pseudo-energy written on the skeleton of the mesh decomposition, which establishes the stability of these methods. Implementation within a finite element solver (GMSH/GetDP) and numerical tests illustrate the theory.
\end{abstract}

Keywords Absorbing Boundary Condition, Domain Decomposition Method, Transmission Condition, Helmholtz equation, Corners, Time-harmonic

\section{Introduction}

The analysis of Domain Decomposition Methods (DDM) [11] for time harmonic wave equations has always received a strong interest from the mathematical community $[9,3,25,10]$ because of its role for the development of efficient linear solvers [7,30]. Optimized DDM $[18,15,5]$ aim at the acceleration of these linear solvers in view of applications, and in most contributions optimized DDM are based on higher order approximations of transmission conditions between subdomains. Recently, the question of DDM acceleration in combination with a sound treatment of mesh singularities, which are unavoidable for meshes obtained from generic softwares, has gained attraction $[16,17,28]$. But as explained in the literature $[16,19,26,28,27]$, optimized DDM and mesh singularities are often in contradiction. Solutions have been proposed $[4,6,14]$ but without a complete

B. Després

Laboratoire Jacques-Louis Lions, Sorbonne Université, 4 place Jussieu, 75005 Paris, France

E-mail: despres@ann.jussieu.fr

A. Nicolopoulos

Laboratoire Jacques-Louis Lions, Sorbonne Université, 4 place Jussieu, 75005 Paris, France

E-mail: nicolopoulos@ljll.math.upmc.fr

B. Thierry

CNRS \& Laboratoire Jacques-Louis Lions, Sorbonne Université, 4 place Jussieu, 75005 Paris, France

E-mail: bertrand.thierry@sorbonne-universite.fr 
mathematical justification. In [25] for example, the optimization is based on quasi-local operators with convenient regularity, but ultimately the problem posed by mesh singularities is not solved. In $[17,19]$, some results have been proven in the purely discrete case. Therefore, there are still many issues widely open for a rigorous mathematical treatment of optimized DDMs with mesh singularities. In particular, we are not aware of any Partial Differential Equations (PDE) based proof of stability, to which convergence follows, for a DDM with corners, either external ones on the boundary of the domain or internal ones on the interface between two subdomains, except the recent contribution [8] in which a completely different approach is used through the multi-trace formalism.

Note that mesh singularities for a polygonal model domain $\Omega \subset \mathbb{R}^{2}$ partitioned in nonoverlapping polygonal subdomains $\Omega_{i}$ can be classified into corners and cross-points. By corners, we understand classical corners, either on the boundary $\partial \Omega$ of the polygonal domain, or on the intersection of the boundaries of two different subdomains $\partial \Omega_{i}$ and $\partial \Omega_{j}$. By cross-points, we understand the general situation of internal points on the intersection of the boundaries of three or more different subdomains $\partial \Omega_{i}, \partial \Omega_{j}, \partial \Omega_{k}, \ldots$

This work contributes to the issue of corners, by studying the mathematical structure of new optimized DDMs with strong stability properties adapted to corners. Cross-points will be treated in subsequent work. The reason for this distinction is that the intuition of our treatment for corners comes naturally from a second order Absorbing Boundary Condition (ABC) with corners, while the generalization to cross-points will require a different algebraic approach outside the scope of this article.

The reference problem of this work is, for the above reasons, the propagation of a timeharmonic wave $u$ in the space $\mathbb{R}^{2}$ generated by a compactly supported source $f$. It is modeled by the Helmholtz equation coupled to the Sommerfeld radiation condition at infinity

$$
\left\{\begin{aligned}
\left(-\Delta-\omega^{2}\right) u & =f, \quad \text { in } \mathbb{R}^{2} \\
\lim _{\|\mathbf{x}\| \rightarrow \infty}\left(\nabla u(\mathbf{x}) \cdot \frac{\mathbf{x}}{\|\mathbf{x}\|}-\mathbf{i} \omega u(\mathbf{x})\right) & =O\left(\frac{1}{\|\mathbf{x}\|^{2}}\right) .
\end{aligned}\right.
$$

To solve (1) using a finite element method, the full propagation domain $\mathbb{R}^{2}$ is truncated. This can be done for example by defining an artificial boundary $\Gamma$ that encloses the support of $f$. In this paper, $\Gamma$ is assumed to be a polygon. This assumption is actually natural as the bounded computational domain $\Omega \subset \mathbb{R}^{2}$, enclosed by $\Gamma=\partial \Omega$, will next be meshed with triangles, making it polygonal anyway. These considerations naturally lead to the Helmholtz equation in a polygonal bounded domain $\Omega$

$$
\left(-\Delta-\omega^{2}\right) u=f
$$

Conditions at the boundary $\Gamma$ of the domain must then be prescribed, such as an $\mathrm{ABC}$ or a combination of ABC with hard, soft or Robin boundary conditions. In this work, we focus on a second order coercive ABC which can be derived from the family described in [13] or in [24]. Let $\mathbf{n}$ and $\mathbf{t}$ be the unit normal and tangential vectors to $\Gamma$ such that the local system $(\mathbf{n}, \mathbf{t})$ is direct. It is well known that the system made of (2) and of the ABC with zero order tangential operator $\partial_{\mathbf{n}} u-\mathbf{i} \omega u=0$ on $\Gamma$ is a well-posed problem in $H^{1}(\Omega)$. The accuracy of this ABC is restricted to first order and higher order conditions yield better approximation properties. A classical second order ABC [23] is $\partial_{\mathbf{n}} u-\mathbf{i} \omega\left(1+\frac{1}{2 \omega^{2}} \partial_{\mathbf{t t}}\right) u=0$. It is based on a second order tangential operator, $\partial_{\mathbf{t t}}$ being the second order tangential derivative along the flat edges of $\Gamma$. In the more general case of a curved boundary, an additional term depending on the curvature radius is introduced [13]. For mathematical reasons, we need a coercive ABC, and this requirement is not satisfied by the operator $u \mapsto\left(1+\frac{1}{2 \omega^{2}} \partial_{\mathbf{t t}}\right) u$. This is why we consider the modified second order ABC that 
writes

$$
\left(1-\frac{1}{2 \omega^{2}} \partial_{\mathbf{t t}}\right) \partial_{\mathbf{n}} u-\mathbf{i} \omega u=0,
$$

and now the operator $\partial_{\mathbf{n}} u \mapsto\left(1-\frac{1}{2 \omega^{2}} \partial_{\mathbf{t t}}\right) \partial_{\mathbf{n}} u$ is formally coercive. This operator is defined on the flat parts of $\Gamma$, that is except at corners. The ABC must thus be complemented with corner conditions $[2,21,22]$. Now that we have introduced these notions, we can describe the original contributions of this work:

- In Section 2, we construct corner conditions to complement the ABC (3). We show that the complemented $\mathrm{ABC}$ has a coercive formulation, and derives various natural inequalities. In particular, a new isometry in Lemma 6 generalizes classical results [9,25]. The well-posedness of the global problem is stated in Theorem 2.

- In Section 3, we construct two different DDMs, named DDM-1 and DDM-2. Both of them solve the Helmholtz equation (2) with the complemented ABC, with various levels of decoupling between the subdomains. As a consequence of the natural inequalities satisfied by the coercive formulation at the boundary, we show in Lemmas 7, 8 and 13 that the DDMs are endowed with a skeleton energy which naturally decreases from one iteration to the other. In this work, this is referred to as the stability of these DDMs: using standard techniques [9,3,25] briefly recalled in Remark 2, the convergence follows.

- In Section 4, we construct a Transmission Condition (TC) for broken-line interfaces. We show in Lemma 10 that the new isometry Lemma 6 for the ABC yields the stability of the new DDM with this TC.

- Some numerical results illustrate the various theoretical properties in our last Section.

- In Appendix A, we explain how to recover curvature effects of the ABC when the polygon tends to a circle.

- In Appendix B, we present DDM-3, an evolution of algorithm DDM-2 with more decoupling.

Conventions. Our constructive method starts from discrete notations adapted to a general mesh. The global indices $k$ and $\ell$ are reserved for the edges of the exterior boundary. The global indices $i$ and $j$ are reserved for subdomains of $\Omega$. The local indices $r$ and $s$ are reserved for the edges of interfaces between two subdomains. Bounds in sums will be omitted or specified depending on the context to help global understanding. With these notations, a node denoted as $\mathbf{A}_{k \ell}^{i j}$ belongs to the intersection of the $k^{\text {th }}$ and of the $\ell^{\text {th }}$ edges of $\Gamma$, and to the boundaries of the $i^{\text {th }}$ and of the $j^{\text {th }}$ subdomains. A subtle distinction will be made by using either the subscripted $\mathbf{n}_{k}$ for the exterior normal to $\Omega$ on the $k^{\text {th }}$ edge of $\Gamma$ or the superscripted $\mathbf{n}^{i}$ for the exterior normal to $\Omega_{i}$. The same distinction will be made between the tangent unit vectors $\mathbf{t}_{k}$ and $\mathbf{t}^{i}$. The index $p$ will be used for algorithm iterations. The purely imaginary number is written in bold $\mathbf{i}^{2}=-1$.

\section{A second order ABC with corner treatment}

\subsection{Geometry and notations}

We define the notations which correspond to the edge and corner geometries illustrated in Figures 1 and 2. On an oriented edge $\Gamma_{k}=\left(\mathbf{a}_{k}, \mathbf{b}_{k}\right)$ of $\Gamma$, the unit tangential vector is $\mathbf{t}_{k}=$ $\frac{\mathbf{b}_{k}-\mathbf{a}_{k}}{\left\|\mathbf{b}_{k}-\mathbf{a}_{k}\right\|}$ and the unit normal vector is $\mathbf{n}_{k}=-\mathbf{t}_{k}^{\perp}$, its rotation of $-\pi / 2$. Table 1 summarizes their coordinates in the local $(x, y)$ frame. At the boundary points $\mathbf{a}_{k}$ and $\mathbf{b}_{k}$ of $\Gamma_{k}$, which are corner points, a unit vector $\tau_{k}$ parallel to $\mathbf{t}_{k}$ and pointing outside of $\Gamma_{k}$ is introduced. 


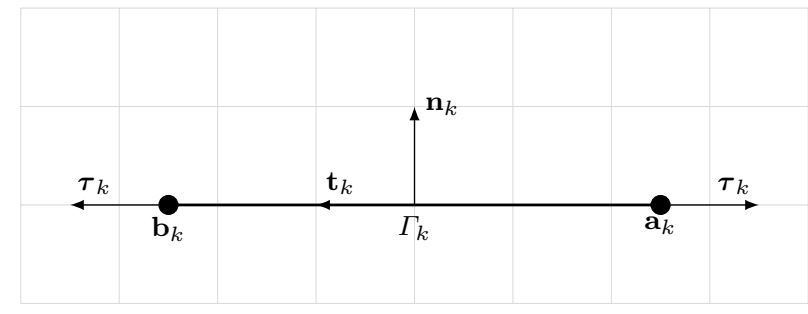

Fig. 1: Edge geometry: normal and tangent vectors $\mathbf{n}_{k}$ and $\mathbf{t}_{k}$, and outgoing corner vectors $\boldsymbol{\tau}_{k}$ on a segment $\Gamma_{k}=\left[\mathbf{a}_{k}, \mathbf{b}_{k}\right]$.

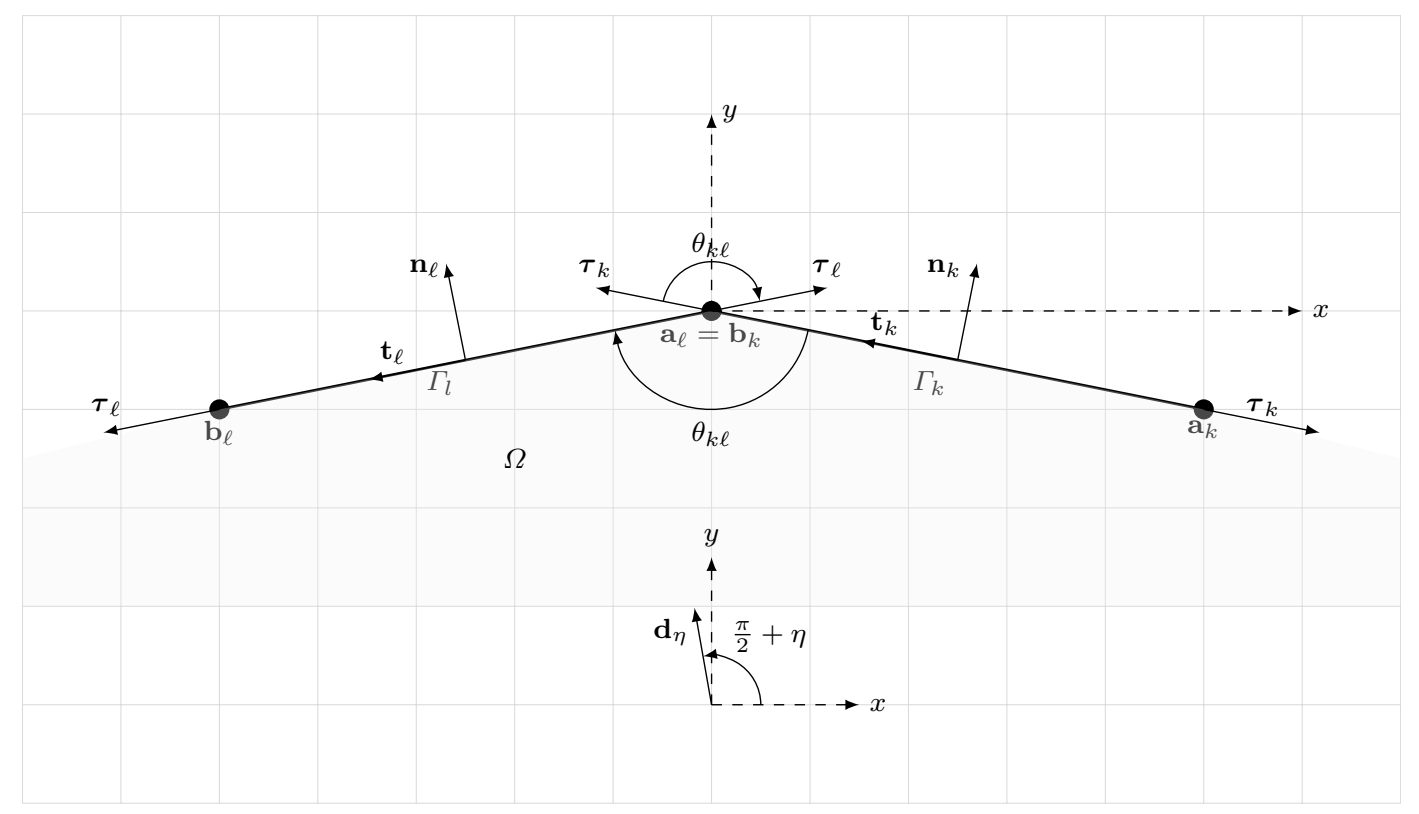

Fig. 2: Corner geometry: local frame at the intersection of two segments $\Gamma_{k}$ and $\Gamma_{\ell}$ with $\mathbf{b}_{k}=$ $\mathbf{a}_{\ell}=\mathbf{A}_{k \ell}$.

With these notations, for two segments $\Gamma_{k}=\left(\mathbf{a}_{k}, \mathbf{b}_{k}\right)$ and $\Gamma_{\ell}=\left(\mathbf{a}_{\ell}, \mathbf{b}_{\ell}\right)$ sharing a common summit $\mathbf{a}_{k}=\mathbf{b}_{\ell}$, we denote this summit

$$
\mathbf{A}_{k \ell}=\mathbf{A}_{\ell k}=\mathbf{a}_{k}=\mathbf{b}_{\ell} .
$$

The chosen convention is to characterize the angle between two such segments by a negative value $\theta_{k \ell} \in(-2 \pi, 0)$, see Figure 2 . The geometrically degenerate case $\theta_{k \ell}=-\pi$ will appear to be a mathematical singularity of some of the formulations in this work. Another singularity will show up for right angles $\theta_{k \ell} \in\left\{-\frac{\pi}{2},-\frac{3 \pi}{2}\right\}$ in other formulations. A rigorous treatment will systematically be indicated for each case, and we suggest the reader to set them aside for the general presentation. 


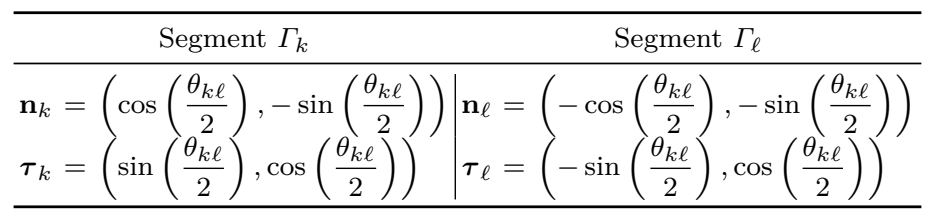

Table 1: Local coordinates of the tangent and normal vectors with respect to the angle $\theta_{k \ell}$ for two intersecting segments $\Gamma_{k}$ and $\Gamma_{\ell}$ at $\mathbf{b}_{k}=\mathbf{a}_{\ell}$.

\subsection{Quasicontinuity relations}

The goal here is to obtain two quasicontinuity relations at a corner point $\mathbf{A}_{k \ell}$ between two segments for a plane wave $u_{\eta}$ with an incident angle $\frac{\pi}{2}+\eta$ as in Figure 2

$$
u_{\eta}(\mathbf{x})=e^{\mathbf{i} \omega\left(\mathbf{d}_{\eta}, \mathbf{x}\right)}, \quad \text { with } \quad \mathbf{d}_{\eta}=\left(\cos \left(\frac{\pi}{2}+\eta\right), \sin \left(\frac{\pi}{2}+\eta\right)\right)=(-\sin (\eta), \cos (\eta)) \in \mathbb{R}^{2} .
$$

At the intersection point $\mathbf{A}_{k \ell}=\mathbf{A}_{\ell k}$, one has the following expressions of the derivatives of $u_{\eta}$

$$
\begin{cases}\partial_{\mathbf{n}_{k}} u_{\eta}\left(\mathbf{A}_{k \ell}\right) & =\mathbf{i} \omega\left(\mathbf{n}_{k}, \mathbf{d}_{\eta}\right) u_{\eta}\left(\mathbf{A}_{k \ell}\right) \\ \partial_{\mathbf{n}_{\ell}} u_{\eta}\left(\mathbf{A}_{k \ell}\right) & =\mathbf{i} \omega\left(\mathbf{n}_{\ell}, \mathbf{d}_{\eta}\right) u_{\eta}\left(\mathbf{A}_{k \ell}\right) \\ \partial_{\boldsymbol{\tau}_{k}} \partial_{\mathbf{n}_{k}} u_{\eta}\left(\mathbf{A}_{k \ell}\right) & =-\omega^{2}\left(\boldsymbol{\tau}_{k}, \mathbf{d}_{\eta}\right)\left(\mathbf{n}_{k}, \mathbf{d}_{\eta}\right) u_{\eta}\left(\mathbf{A}_{k \ell}\right) \\ \partial_{\boldsymbol{\tau}_{\ell}} \partial_{\mathbf{n}_{\ell}} u_{\eta}\left(\mathbf{A}_{k \ell}\right) & =-\omega^{2}\left(\boldsymbol{\tau}_{\ell}, \mathbf{d}_{\eta}\right)\left(\mathbf{n}_{\ell}, \mathbf{d}_{\eta}\right) u_{\eta}\left(\mathbf{A}_{k \ell}\right)\end{cases}
$$

The scalar products between the direction $\mathbf{d}_{\eta}$ and the normal and tangent vectors of $\Gamma_{k}$ are

$$
\left\{\begin{array}{l}
\left(\mathbf{n}_{k}, \mathbf{d}_{\eta}\right)=-\cos \left(\frac{\theta_{k \ell}}{2}\right) \sin (\eta)-\sin \left(\frac{\theta_{k \ell}}{2}\right) \cos (\eta)=-\sin \left(\frac{\theta_{k \ell}}{2}+\eta\right), \\
\left(\boldsymbol{\tau}_{k}, \mathbf{d}_{\eta}\right)=-\sin \left(\frac{\theta_{k \ell}}{2}\right) \sin (\eta)+\cos \left(\frac{\theta_{k \ell}}{2}\right) \cos (\eta)=\cos \left(\frac{\theta_{k \ell}}{2}+\eta\right),
\end{array}\right.
$$

while the scalar products between the direction $\mathbf{d}_{\eta}$ and the normal and tangent vectors of $\Gamma_{\ell}$ are

$$
\left\{\begin{array}{l}
\left(\mathbf{n}_{\ell}, \mathbf{d}_{\eta}\right)=\cos \left(\frac{\theta_{k \ell}}{2}\right) \sin (\eta)-\sin \left(\frac{\theta_{k \ell}}{2}\right) \cos (\eta)=-\sin \left(\frac{\theta_{k \ell}}{2}-\eta\right) \\
\left(\boldsymbol{\tau}_{\ell}, \mathbf{d}_{\eta}\right)=\sin \left(\frac{\theta_{k \ell}}{2}\right) \sin (\eta)+\cos \left(\frac{\theta_{k \ell}}{2}\right) \cos (\eta)=\cos \left(\frac{\theta_{k \ell}}{2}-\eta\right)
\end{array}\right.
$$

For small $\eta$, we outline two linear combinations of $u_{\eta}$ derivatives (4). We will use these linear combinations to define quasicontinuity relations.

Lemma 1 Consider a corner point $\mathbf{A}_{k \ell}$. For $\eta$ close to zero, the following relation holds

$$
\mathbf{i} \omega \cos \left(\theta_{k \ell}\right)\left(\partial_{\mathbf{n}_{k}} u_{\eta}-\partial_{\mathbf{n}_{\ell}} u_{\eta}\right)\left(\mathbf{A}_{k \ell}\right)-\cos \left(\frac{\theta_{k \ell}}{2}\right)\left(\partial_{\boldsymbol{\tau}_{k}} \partial_{\mathbf{n}_{k}} u_{\eta}-\partial_{\boldsymbol{\tau}_{\ell}} \partial_{\mathbf{n}_{\ell}} u_{\eta}\right)\left(\mathbf{A}_{k \ell}\right)=O\left(\eta^{3}\right) \text {. }
$$

Proof On the one hand, the difference between the first order derivatives from (4) is

$$
\begin{aligned}
\left(\partial_{\mathbf{n}_{k}} u_{\eta}-\partial_{\mathbf{n}_{\ell}} u_{\eta}\right)\left(\mathbf{A}_{k \ell}\right) & =\mathbf{i} \omega\left[-\sin \left(\frac{\theta_{k \ell}}{2}+\eta\right)+\sin \left(\frac{\theta_{k \ell}}{2}-\eta\right)\right] u_{\eta}\left(\mathbf{A}_{k \ell}\right) \\
& =-2 \mathbf{i} \omega \eta \cos \left(\frac{\theta_{k \ell}}{2}\right) u_{\eta}\left(\mathbf{A}_{k \ell}\right)+O\left(\eta^{3}\right) .
\end{aligned}
$$


On the other hand, the difference between the second order derivatives from (4) is

$$
\begin{aligned}
& \left(\partial_{\boldsymbol{\tau}_{k}} \partial_{\mathbf{n}_{k}} u_{\eta}-\partial_{\boldsymbol{\tau}_{\ell}} \partial_{\mathbf{n}_{\ell}} u_{\eta}\right)\left(\mathbf{A}_{k \ell}\right) \\
& =-\omega^{2}\left[-\cos \left(\frac{\theta_{k \ell}}{2}+\eta\right) \sin \left(\frac{\theta_{k \ell}}{2}+\eta\right)+\cos \left(\frac{\theta_{k \ell}}{2}-\eta\right) \sin \left(\frac{\theta_{k \ell}}{2}-\eta\right)\right] u_{\eta}\left(\mathbf{A}_{k \ell}\right) \\
& =-\omega^{2}\left[-\frac{1}{2} \sin \left(\theta_{k \ell}+2 \eta\right)+\frac{1}{2} \sin \left(\theta_{k \ell}-2 \eta\right)\right] u_{\eta}\left(\mathbf{A}_{k \ell}\right) \\
& =-\omega^{2}\left[-2 \cos \left(\theta_{k \ell}\right)+O\left(\eta^{3}\right)\right] u_{\eta}\left(\mathbf{A}_{k \ell}\right)=2 \omega^{2} \eta \cos \left(\theta_{k \ell}\right) u_{\eta}\left(\mathbf{A}_{k \ell}\right)+O\left(\eta^{3}\right) .
\end{aligned}
$$

Combining these two relations yields the claim.

Lemma 2 For a corner point $\mathbf{A}_{k \ell}$ and $\eta$ close to zero, the following relation holds

$$
-\mathbf{i} \omega \cos \left(\frac{\theta_{k \ell}}{2}\right)\left(\partial_{\mathbf{n}_{k}} u_{\eta}+\partial_{\mathbf{n}_{\ell}} u_{\eta}\right)\left(\mathbf{A}_{k \ell}\right)+\left(\partial_{\boldsymbol{\tau}_{k}} \partial_{\mathbf{n}_{k}} u_{\eta}+\partial_{\boldsymbol{\tau}_{\ell}} \partial_{\mathbf{n}_{\ell}} u_{\eta}\right)\left(\mathbf{A}_{k \ell}\right)=O\left(\eta^{2}\right) .
$$

Proof First, adding the two first order derivatives from (4) gives

$$
\begin{aligned}
\left(\partial_{\mathbf{n}_{k}} u_{\eta}+\partial_{\mathbf{n}_{\ell}} u_{\eta}\right)\left(\mathbf{A}_{k \ell}\right) & =-\mathbf{i} \omega\left[\sin \left(\frac{\theta_{k \ell}}{2}+\eta\right)+\sin \left(\frac{\theta_{k \ell}}{2}-\eta\right)\right] u_{\eta}\left(\mathbf{A}_{k \ell}\right) \\
& =-2 \mathbf{i} \omega \sin \left(\frac{\theta_{k \ell}}{2}\right) \cos (\eta) u_{\eta}\left(\mathbf{A}_{k \ell}\right) \\
& =-2 \mathbf{i} \omega \sin \left(\frac{\theta_{k \ell}}{2}\right) u_{\eta}\left(\mathbf{A}_{k \ell}\right)+O\left(\eta^{2}\right) .
\end{aligned}
$$

Second, adding the two second order derivatives from (4) gives

$$
\begin{aligned}
& \left(\partial_{\boldsymbol{\tau}_{k}} \partial_{\mathbf{n}_{k}} u_{\eta}+\partial_{\boldsymbol{\tau}_{\ell}} \partial_{\mathbf{n}_{\ell}} u_{\eta}\right)\left(\mathbf{A}_{k \ell}\right) \\
& \quad=\omega^{2}\left[\sin \left(\frac{\theta_{k \ell}}{2}+\eta\right) \cos \left(\frac{\theta_{k \ell}}{2}+\eta\right)+\sin \left(\frac{\theta_{k \ell}}{2}-\eta\right) \cos \left(\frac{\theta_{k \ell}}{2}-\eta\right)\right] u_{\eta}\left(\mathbf{A}_{k \ell}\right) \\
& \quad=\omega^{2}\left[\frac{1}{2} \sin \left(\theta_{k \ell}+2 \eta\right)+\frac{1}{2} \sin \left(\theta_{k \ell}-2 \eta\right)\right] u_{\eta}\left(\mathbf{A}_{k \ell}\right) \\
& \quad=\omega^{2} \sin \left(\theta_{k \ell}\right) u_{\eta}\left(\mathbf{A}_{k \ell}\right)+O\left(\eta^{2}\right) \\
& \quad=2 \omega^{2} \sin \left(\theta_{k \ell} / 2\right) \cos \left(\frac{\theta_{k \ell}}{2}\right) u_{\eta}\left(\mathbf{A}_{k \ell}\right)+O\left(\eta^{2}\right) .
\end{aligned}
$$

The expected relation is obtained by combining the above two equations.

We now introduce the quantity $\varphi_{k}:=\frac{1}{\mathbf{i} \omega} \partial_{\mathbf{n}_{k}} u_{\eta}$ on each segment $\Gamma_{k}$. Rewriting (5) and (6) using this new variable and dropping the $O\left(\eta^{2}\right)$ and $O\left(\eta^{3}\right)$ terms leads to the second order quasicontinuity relations

$$
\left\{\begin{array}{r}
\mathbf{i} \omega \cos \left(\theta_{k \ell}\right)\left(\varphi_{k}-\varphi_{\ell}\right)\left(\mathbf{A}_{k \ell}\right)-\cos \left(\frac{\theta_{k \ell}}{2}\right)\left(\partial_{\boldsymbol{\tau}_{k}} \varphi_{k}-\partial_{\boldsymbol{\tau}_{\ell}} \varphi_{\ell}\right)\left(\mathbf{A}_{k \ell}\right)=0 \\
-\mathbf{i} \omega \cos \left(\frac{\theta_{k \ell}}{2}\right)\left(\varphi_{k}+\varphi_{\ell}\right)\left(\mathbf{A}_{k \ell}\right)+\left(\partial_{\boldsymbol{\tau}_{k}} \varphi_{k}+\partial_{\boldsymbol{\tau}_{\ell}} \varphi_{\ell}\right)\left(\mathbf{A}_{k \ell}\right)=0
\end{array}\right.
$$

For a flat angle $\theta_{k \ell}=-\pi$, this system corresponds exactly to the continuity relations

$$
\left\{\begin{aligned}
\varphi_{k}\left(\mathbf{A}_{k \ell}\right)-\varphi_{\ell}\left(\mathbf{A}_{k \ell}\right) & =0 \\
\partial_{\boldsymbol{\tau}_{k}} \varphi_{k}\left(\mathbf{A}_{k \ell}\right)+\partial_{\boldsymbol{\tau}_{\ell}} \varphi_{\ell}\left(\mathbf{A}_{k \ell}\right) & =0
\end{aligned}\right.
$$

We now recast these equations to have symmetric relations with repect to $k$ and $\ell$. A first symmetric system of quasicontinuity relations is derived for non-flat corners. 
Lemma 3 Consider a corner point $\mathbf{A}_{k \ell}$ such that $\theta_{k \ell} \neq-\pi$. If relations ( 7$)$ are satisfied then the following second order quasicontinuity relations are also satisfied

$$
\left\{\begin{array}{c}
\partial_{\boldsymbol{\tau}_{k}} \varphi_{k}\left(\mathbf{A}_{k \ell}\right)-\mathbf{i} \frac{\omega}{2}\left(\frac{\cos \left(\theta_{k \ell}\right)}{\cos \left(\frac{\theta_{k \ell}}{2}\right)}+\cos \left(\frac{\theta_{k \ell}}{2}\right)\right) \varphi_{k}\left(\mathbf{A}_{k \ell}\right) \\
=\mathbf{i} \frac{\omega}{2}\left(-\frac{\cos \left(\theta_{k \ell}\right)}{\cos \left(\frac{\theta_{k \ell}}{2}\right)}+\cos \left(\frac{\theta_{k \ell}}{2}\right)\right) \varphi_{\ell}\left(\mathbf{A}_{k \ell}\right), \\
\partial_{\tau_{\ell}} \varphi_{\ell}\left(\mathbf{A}_{k \ell}\right)-\mathbf{i} \frac{\omega}{2}\left(\frac{\cos \left(\theta_{\ell k}\right)}{\cos \left(\frac{\theta_{\ell k}}{2}\right)}+\cos \left(\frac{\theta_{\ell k}}{2}\right)\right) \varphi_{\ell}\left(\mathbf{A}_{k \ell}\right) \\
=\mathbf{i} \frac{\omega}{2}\left(-\frac{\cos \left(\theta_{\ell k}\right)}{\cos \left(\frac{\theta_{\ell k}}{2}\right)}+\cos \left(\frac{\theta_{\ell k}}{2}\right)\right) \varphi_{k}\left(\mathbf{A}_{k \ell}\right) .
\end{array}\right.
$$

Proof For $\theta_{k \ell} \neq-\pi$, system (7) is equivalent to

$$
\left\{\begin{array}{c}
-\mathbf{i} \omega \frac{\cos \left(\theta_{k \ell}\right)}{\cos \left(\frac{\theta_{k \ell}}{2}\right)}\left(\varphi_{k}-\varphi_{\ell}\right)\left(\mathbf{A}_{k \ell}\right)+\left(\partial_{\boldsymbol{\tau}_{k}} \varphi_{k}-\partial_{\boldsymbol{\tau}_{\ell}} \varphi_{\ell}\right)\left(\mathbf{A}_{k \ell}\right)=0 \\
-\mathbf{i} \omega \cos \left(\frac{\theta_{k \ell}}{2}\right)\left(\varphi_{k}+\varphi_{\ell}\right)\left(\mathbf{A}_{k \ell}\right)+\left(\partial_{\boldsymbol{\tau}_{k}} \varphi_{k}+\partial_{\boldsymbol{\tau}_{\ell}} \varphi_{\ell}\right)\left(\mathbf{A}_{k \ell}\right)=0
\end{array}\right.
$$

The first relation of (8) is obtained by adding these two lines and dividing by 2 , and the second one is obtained by subtracting the first line to the second and dividing by 2 .

A family of mixed symmetric systems of quasicontinuity relations parametrized by a coefficient $\beta \neq 0$ can also be constructed, for non right angled corners.

Lemma 4 Consider a corner point $\mathbf{A}_{k \ell}$ such that $\theta_{k \ell} \notin\left\{-\frac{\pi}{2},-\frac{3 \pi}{2}\right\}$ and let $\beta \neq 0$. If relations (7) are satisfied then the following second order quasicontinuity relations are also satisfied

$$
\left\{\begin{array}{l}
\left(1+\beta \frac{\mathbf{i} \cos \left(\frac{\theta_{k \ell}}{2}\right)}{\omega \cos \left(\theta_{k \ell}\right)}\right) \partial_{\boldsymbol{\tau}_{k}} \varphi_{k}\left(\mathbf{A}_{k \ell}\right)+\left(\beta-\mathbf{i} \omega \cos \left(\frac{\theta_{k \ell}}{2}\right)\right) \varphi_{k}\left(\mathbf{A}_{k \ell}\right) \\
=\left(-1+\beta \frac{\mathbf{i} \cos \left(\frac{\theta_{k \ell}}{2}\right)}{\omega \cos \left(\theta_{k \ell}\right)}\right) \partial_{\boldsymbol{\tau}_{\ell}} \varphi_{\ell}\left(\mathbf{A}_{k \ell}\right)+\left(\beta+\mathbf{i} \omega \cos \left(\frac{\theta_{k \ell}}{2}\right)\right) \varphi_{\ell}\left(\mathbf{A}_{k \ell}\right) \\
\left(1+\beta \frac{\mathbf{i} \cos \left(\frac{\theta_{\ell k}}{2}\right)}{\omega \cos \left(\theta_{\ell k}\right)}\right) \partial_{\boldsymbol{\tau}_{\ell}} \varphi_{\ell}\left(\mathbf{A}_{k \ell}\right)+\left(\beta-\mathbf{i} \omega \cos \left(\frac{\theta_{\ell k}}{2}\right)\right) \varphi_{\ell}\left(\mathbf{A}_{k \ell}\right) \\
=\left(-1+\beta \frac{\mathbf{i} \cos \left(\frac{\theta_{\ell k}}{2}\right)}{\omega \cos \left(\theta_{\ell k}\right)}\right) \partial_{\boldsymbol{\tau}_{k}} \varphi_{k}\left(\mathbf{A}_{k \ell}\right)+\left(\beta+\mathbf{i} \omega \cos \left(\frac{\theta_{\ell k}}{2}\right)\right) \varphi_{k}\left(\mathbf{A}_{k \ell}\right)
\end{array}\right.
$$

Proof Multiplying the first line of $(7)$ by $\frac{\beta}{\mathrm{i} \omega \cos \left(\theta_{k \ell}\right)}$, and adding it to the second line leads to the first relation of (9). Multiplying by $\frac{-\beta}{\mathrm{i} \omega \cos \left(\theta_{k \ell}\right)}$ instead gives the second relation. This algebra is non singular for $\beta \neq 0$.

Desingularization near $\cos \left(\theta_{k \ell}\right)=0$ and homogeneity considerations lead to take $\beta$ proportional to $\omega \cos \left(\theta_{k \ell}\right)$. For energy reasons explained in the sequel (see Remark 4), the sign of $\beta$ will also prescribed. Therefore, we immediately set

$$
\beta:=-|\omega| \cos \left(\theta_{k \ell}\right) \text {. }
$$

We will now consider relations (9) for any angle $\theta_{k \ell}$ : when $\theta_{k \ell}=-\pi / 2$ or $-3 \pi / 2$, the relations will be desingularized beforehand using the expression of $\beta$. We note here that there exists previous works $[23,28]$ treating the specific case of right angles, for which $\mathbf{n}_{k}$ and $\mathbf{t}_{\ell}$ are colinear as well as $\mathbf{t}_{k}$ and $\mathbf{n}_{\ell}$. 


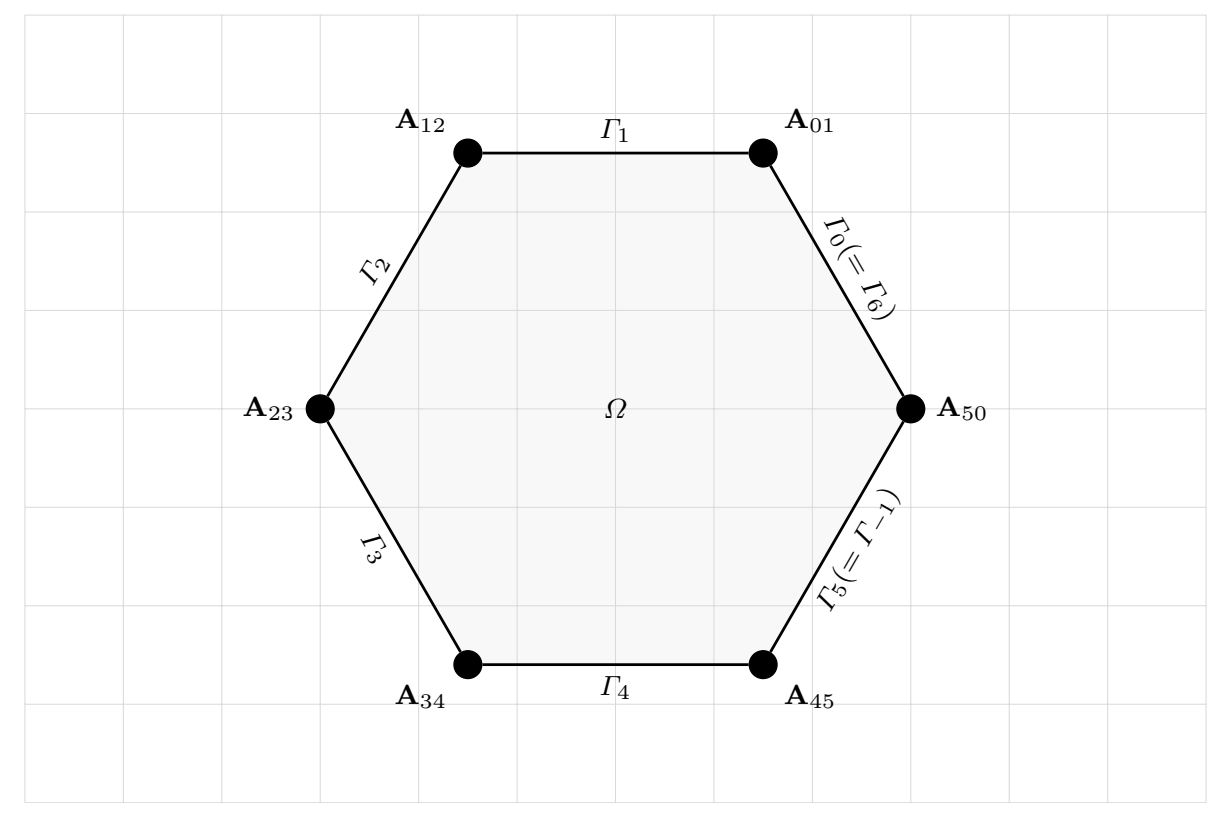

Fig. 3: Example of a polygonal domain $\Omega$ with boundary $\Gamma=\cup_{k=0}^{N-1} \Gamma_{k}$ composed of 6 segments.

\subsection{Definition of the second order $\mathrm{ABC}$}

The boundary $\Gamma:=\overline{\bigcup_{k=0}^{K-1} \Gamma_{k}}$ is assumed to be polygonal and decomposed in $K \geq 3$ segments, as illustrated in Figure 3, with $\Gamma_{K}=\Gamma_{0}$ and $\Gamma_{-1}=\Gamma_{K-1}$. The segments are numbered consecutively counter-clockwise and the previous notations are used: on a segment $\Gamma_{k}$, the normal vector $\mathbf{n}_{k}$ is pointing outside the domain $\Omega$; the tangential vector $\mathbf{t}_{k}$ points towards $\Gamma_{k+1}$; and at the two corners $\mathbf{A}_{k \ell}$, for $\ell=k \pm 1$, the vectors $\boldsymbol{\tau}_{k}\left(\mathbf{A}_{k \ell}\right)$ are tangent to $\Gamma_{k}$ and pointing outside.

For $u \in L^{2}(\Gamma)$, we define $\varphi \in \oplus_{k} H^{1}\left(\Gamma_{k}\right)$ such that, for $k=0, \ldots, K-1$, the quantity $\varphi_{k}=\left.\varphi\right|_{\Gamma_{k}}$ is the variational solution of

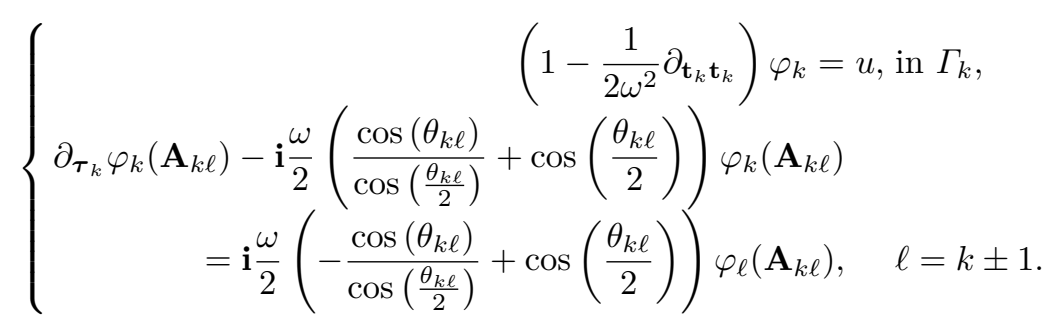

Let $\varphi \in \oplus_{k} H^{1}\left(\Gamma_{k}\right)$ be a strong solution of (11). Integrating by parts the equation on each $\Gamma_{k}$ against the conjugate of $\psi \in \oplus_{k} H^{1}\left(\Gamma_{k}\right)$ gives the variational form

$$
\sum_{k}\left[\int_{\Gamma_{k}}\left(\varphi_{k} \overline{\psi_{k}}+\frac{1}{2 \omega^{2}} \frac{\partial \varphi_{k}}{\partial \mathbf{t}_{k}} \overline{\frac{\partial \psi_{k}}{\partial \mathbf{t}_{k}}}\right) \mathrm{d} \gamma-\frac{1}{2 \omega^{2}} \sum_{\ell=k \pm 1}\left(\frac{\partial \varphi_{k}}{\partial \boldsymbol{\tau}_{k}} \overline{\psi_{k}}\right)\left(\mathbf{A}_{k \ell}\right)\right]=\sum_{k} \int_{\Gamma_{k}} u \overline{\psi_{k}} \mathrm{~d} \gamma
$$


The boundary $\Gamma$ being closed, the corner terms can be regrouped, yielding

$$
\sum_{k} \sum_{\ell=k \pm 1}\left(\frac{\partial \varphi_{k}}{\partial \boldsymbol{\tau}_{k}} \overline{\psi_{k}}\right)\left(\mathbf{A}_{k \ell}\right)=\sum_{\substack{k \\ \ell=k+1}}\left(\frac{\partial \varphi_{k}}{\partial \boldsymbol{\tau}_{k}} \overline{\psi_{k}}+\frac{\partial \varphi_{\ell}}{\partial \boldsymbol{\tau}_{\ell}} \overline{\psi_{\ell}}\right)\left(\mathbf{A}_{k \ell}\right) .
$$

The quasicontinuity relations (8) verified by $\varphi$ ensure that at $\mathbf{A}_{k \ell}$, one has

$$
\begin{aligned}
\frac{\partial \varphi_{k}}{\partial \boldsymbol{\tau}_{k}} \overline{\psi_{k}}+\frac{\partial \varphi_{\ell}}{\partial \boldsymbol{\tau}_{\ell}} \overline{\psi_{\ell}} & =\frac{1}{2}\left(\frac{\partial \varphi_{k}}{\partial \boldsymbol{\tau}_{k}}+\frac{\partial \varphi_{\ell}}{\partial \boldsymbol{\tau}_{\ell}}\right)\left(\overline{\psi_{k}}+\overline{\psi_{\ell}}\right)+\frac{1}{2}\left(\frac{\partial \varphi_{k}}{\partial \boldsymbol{\tau}_{k}}-\frac{\partial \varphi_{\ell}}{\partial \boldsymbol{\tau}_{\ell}}\right)\left(\overline{\psi_{k}}-\overline{\psi_{\ell}}\right) \\
& =\frac{\mathbf{i} \omega}{2}\left[\cos \left(\frac{\theta_{k \ell}}{2}\right)\left(\varphi_{k}+\varphi_{\ell}\right)\left(\overline{\psi_{k}}+\overline{\psi_{\ell}}\right)+\frac{\cos \left(\theta_{k \ell}\right)}{\cos \left(\frac{\theta_{k \ell}}{2}\right)}\left(\varphi_{k}-\varphi_{\ell}\right)\left(\overline{\psi_{k}}-\overline{\psi_{\ell}}\right)\right]
\end{aligned}
$$

After substitution in (12), the variational formulation reads

$$
\left\{\begin{array}{l}
\text { Find } \varphi \in \oplus_{k} H^{1}\left(\Gamma_{k}\right) \text { such that for every test function } \psi \in \oplus_{k} H^{1}\left(\Gamma_{k}\right), \\
a(\varphi, \psi)=\sum_{k} \int_{\Gamma_{k}} u \overline{\psi_{k}} \mathrm{~d} \gamma
\end{array}\right.
$$

where, for $(\varphi, \psi) \in \oplus_{k} H^{1}\left(\Gamma_{k}\right) \times \oplus_{k} H^{1}\left(\Gamma_{k}\right)$, the sesquilinear form is

$$
\begin{aligned}
a(\varphi, \psi)= & \sum_{k} \int_{\Gamma_{k}}\left(\varphi_{k} \overline{\psi_{k}}+\frac{1}{2 \omega^{2}} \frac{\partial \varphi_{k}}{\partial \mathbf{t}_{k}} \overline{\frac{\partial \psi_{k}}{\partial \mathbf{t}_{k}}}\right) \mathrm{d} \gamma \\
& -\frac{\mathbf{i}}{4 \omega} \sum_{\substack{k \\
\ell=k+1}}\left(\cos \left(\frac{\theta_{k \ell}}{2}\right)\left(\varphi_{k}+\varphi_{\ell}\right)\left(\overline{\psi_{k}}+\overline{\psi_{\ell}}\right)+\frac{\cos \left(\theta_{k \ell}\right)}{\cos \left(\frac{\theta_{k \ell}}{2}\right)}\left(\varphi_{k}-\varphi_{\ell}\right)\left(\overline{\psi_{k}}-\overline{\psi_{\ell}}\right)\right)\left(\mathbf{A}_{k \ell}\right) .
\end{aligned}
$$

Theorem 1 For $u \in L^{2}(\Gamma)$, there exists a unique solution $\varphi \in \oplus_{k} H^{1}\left(\Gamma_{k}\right)$ of the variational formulation (13).

Proof The well posedness of problem (13) amounts to show that a given system of linear ordinary differential equations admits a unique solution: since it is ODE based, it can be reduced to a finite dimensional system, and it is sufficient to prove uniqueness of the solution to obtain wellposedness.

Take $\psi=\phi$ in (13). The real part verifies

$$
\sum_{k} \int_{\Gamma_{k}}\left(\left|\varphi_{k}\right|^{2}+\frac{1}{2 \omega^{2}}\left|\frac{\partial \varphi_{k}}{\partial \mathbf{t}_{k}}\right|^{2}\right) \mathrm{d} \gamma=\operatorname{Re}\left(\sum_{k} \int_{\Gamma_{k}} u \overline{\varphi_{k}} \mathrm{~d} \gamma\right)
$$

implying that

$$
\|\varphi\|_{L^{2}(\Gamma)}^{2}+\frac{1}{2 \omega^{2}} \sum_{k}\left\|\frac{\partial \varphi_{k}}{\partial \mathbf{t}_{k}}\right\|_{L^{2}\left(\Gamma_{k}\right)}^{2} \leq\|\varphi\|_{L^{2}(\Gamma)}\|u\|_{L^{2}(\Gamma)} .
$$

Taking $u=0$ leads to $\varphi=0$, which proves the uniqueness of the variational solution for $u \in$ $L^{2}(\Gamma)$, and therefore its existence.

We now define the operator

$$
T: u \in L^{2}(\Gamma) \longmapsto T u=\varphi \in L^{2}(\Gamma)
$$

where $\varphi$ is the unique solution to (13) for a given $u \in L^{2}(\Gamma)$. 
Proposition 1 The operator $T$ satisfies the bound $\|T\|_{\mathcal{L}\left(L^{2}(\Gamma)\right)} \leq 1$.

Proof For $u \in L^{2}(\Gamma)$ and $\varphi=T u \in \oplus_{k} H^{1}\left(\Gamma_{k}\right) \subset L^{2}(\Gamma)$, one has

$$
(T u, T u)_{L^{2}(\Gamma)}=\sum_{k} \int_{\Gamma_{k}}\left(\frac{-1}{2 \omega^{2}}\left|\frac{\partial \varphi_{k}}{\partial \mathbf{t}_{k}}\right|^{2}+\operatorname{Re}\left(u \overline{\varphi_{k}}\right)\right) \mathrm{d} \gamma \leq \operatorname{Re}(u, T u)_{L^{2}(\Gamma)}
$$

and consequently $\|T\|_{\mathcal{L}\left(L^{2}(\Gamma)\right)}=\inf _{u \in L^{2}(\Gamma)} \frac{\|T u\|_{L^{2}(\Gamma)}}{\|u\|_{L^{2}(\Gamma)}} \leq 1$.

Remark 1 Starting from the sesquilinear form $a^{*}(\varphi, \psi)=\overline{a(\psi, \varphi)}$, one defines in a similar way an operator $S$. It is a standard property that $S=T^{*}$ is the adjoint operator of $T$ with respect to space $L^{2}(\Gamma)$. Indeed, one has by definition that $a(T u, S v)=(u, S v)_{L^{2}(\Gamma)}$ and that $a^{*}(S v, T u)=$ $(v, T u)_{L^{2}(\Gamma)}$. Therefore, for all $u, v \in L^{2}(\Gamma)$

$$
(T u, v)_{L^{2}(\Gamma)}=\overline{(v, T u)_{L^{2}(\Gamma)}}=\overline{a^{*}(S v, T u)}=a(T u, S v)=(u, S v)_{L^{2}(\Gamma)}
$$

which shows that $S=T^{*}$ as an $L^{2}(\Gamma)$ operator.

The operator $T$ is now used as a second order ABC for the Helmholtz equation (2) to obtain

$$
\left\{\begin{array}{l}
\left(-\Delta-\omega^{2}\right) u=f \text { in } \Omega, \\
\partial_{\mathbf{n}} u-\mathbf{i} \omega T u=g \text { on } \Gamma .
\end{array}\right.
$$

The associated weak formulation writes

$$
\left\{\begin{array}{l}
\text { Find } u \in H^{1}(\Omega) \text { such that for all } v \in H^{1}(\Omega) \\
\int_{\Omega}\left(\nabla u \cdot \nabla \bar{v}-\omega^{2} u \bar{v}\right) \mathrm{d} \mathbf{x}-\mathbf{i} \omega \int_{\Gamma} T u \bar{v} \mathrm{~d} \gamma=\int_{\Omega} f \bar{v} \mathrm{~d} \mathbf{x}+\int_{\Gamma} g \bar{v} \mathrm{~d} \gamma .
\end{array}\right.
$$

Theorem 2 There exists a unique solution to the variational problem (17).

Proof Classical methods based on a coercive plus compact decomposition, Fredholm alternative, and a unique continuation principle, yield the existence and uniqueness of the variational solution $u \in H^{1}(\Omega)$. The key step is the uniqueness of the solution. For $f=0$ and $g=0$ in (17), inequality (15) yields $T u=0$ and consequently, due to (16), the normal derivative $\partial_{\mathbf{n}} u$ also vanishes. Moreover, the variational definition (13) of $\varphi=T u$ implies that $u$ actually vanishes on $\Gamma$. Since $\partial_{\mathbf{n}} u=0$ and $u=0$ on $\Gamma$, one can invoke a unique continuation principle to get $u=0$ in $\Omega$. Technical details are left to the reader.

\subsection{Two technical lemmas}

We detail two lemmas which summarize important properties of the operator $T$, that will be of substantial importance in Section 4.

Lemma 5 The operator $T+T^{*}$ is self-adjoint and positive.

Proof The self-adjoint property is straightforward. Let us now show the positiveness. For any $u \in L^{2}(\Gamma)$, the following relation holds

$$
\begin{aligned}
\left(\left(T+T^{*}\right) u, u\right)_{L^{2}(\Gamma)}=2 \operatorname{Re}(u, T u)_{L^{2}(\Gamma)} & =2 \operatorname{Re}[a(T u, T u)] \\
& =2\left(\|T u\|_{L^{2}(\Gamma)}^{2}+\frac{1}{2 \omega^{2}} \sum_{k}\left\|\partial_{\mathbf{t}_{k}} T u\right\|_{L^{2}(\Gamma)}^{2}\right) \geq 0 .
\end{aligned}
$$


If $u \in L^{2}(\Gamma)$ is such that $\left(T+T^{*}\right) u=0$, then necessarily $T u=0$ and, for all $\psi \in \oplus_{k} H^{1}\left(\Gamma_{k}\right)$

$$
0=a(T u, \psi)=(u, \psi)_{L^{2}(\Gamma)},
$$

which implies that $u=0$.

The operator $T+T^{*}$ is also compact in $L^{2}(\Gamma)$, and therefore admits a spectral decomposition $\left(T+T^{*}\right) u_{n}=\lambda_{n} u_{n},\left\|u_{n}\right\|_{L^{2}(\Gamma)}=1$, where $\operatorname{span}\left\{u_{n}\right\}=L^{2}(\Gamma)$. The eigenvalues satisfy $2 \geq \lambda_{n} \geq$ $\lambda_{n+1}>0$ and $\lambda_{n}$ converges towards zero as $n$ goes to infinity. This leads us to the definition of the $L^{2}(\Gamma)$ subspace

equipped with the norm

$$
H_{T}^{1}(\Gamma):=\left\{g \in L^{2}(\Gamma),\|g\|<\infty\right\},
$$

$$
\|g\|^{2}:=\sum_{n \geq 0} \frac{\left|g_{n}\right|^{2}}{\lambda_{n}}, \quad \text { with } \quad g_{n}=\left(g, u_{n}\right)_{L^{2}(\Gamma)} .
$$

For $g \in H_{T}^{1}(\Gamma)$, one has $\|g\|^{2}=\left\|\left(T+T^{*}\right)^{-1 / 2} g\right\|_{L^{2}(\Gamma)}^{2}$. The scalar product associated with the norm $\|\cdot\| \cdot \|$ is denoted by $(g, h)_{\|\cdot\| \|}=\sum_{n} \frac{g_{n} \overline{h_{n}}}{\lambda_{n}}$.

Definition 1 Let $\mathcal{T}: L^{2}(\Gamma) \rightarrow L^{2}(\Gamma)$ be the operator such that for $g \in L^{2}(\Gamma)$,

$$
\mathcal{T} g=\partial_{\mathbf{n}} u+\mathbf{i} \omega T^{*} u,
$$

where $u$ is the weak solution of (16) with $f=0$.

The following lemma describes an interesting algebraic property which generalizes the classical one for $T=I[9,3]$ and the generalized one where $T$ is quasilocal with convenient regularity [25]. The algebraic property has the form of an isometry lemma which fundamentally expresses that a specific norm of $\partial_{\mathbf{n}} u+\mathbf{i} \omega T^{*} u$ is equal to the one of $\partial_{\mathbf{n}} u-\mathbf{i} \omega T u$. It can be interpreted as a generalized scattering identity for operators $T \neq T^{*}$.

Lemma 6 For all $g \in H_{T}^{1}(\Gamma)$, one has the identity $\|g\|=\|\mathcal{T} g\|$.

Proof First we show that $\mathcal{T}$ is a bounded operator in $H_{T}^{1}(\Gamma)$. Let $u \in H^{1}(\Omega)$ be the weak solution of (16) with $f=0$ and $g \in H_{T}^{1}(\Gamma)$. It follows that

$$
\mathcal{T} g=\partial_{\mathbf{n}} u+\mathbf{i} \omega T^{*} u=g+\mathbf{i} \omega\left(T+T^{*}\right) u .
$$

The quantity $\left(T+T^{*}\right) u$ belongs to $H_{T}^{1}(\Gamma)$ since we have

$$
\left.\left\|\left(T+T^{*}\right) u\right\|\right|^{2}=\sum_{n \geq 0} \frac{1}{\lambda_{n}}\left|\left(\left(T+T^{*}\right) u, u_{n}\right)_{L^{2}(\Gamma)}\right|^{2}=\sum_{n \geq 0} \frac{1}{\lambda_{n}}\left|\left(u, \lambda_{n} u_{n}\right)_{L^{2}(\Gamma)}\right|^{2}=\sum_{n \geq 0} \lambda_{n}\left|\left(u, u_{n}\right)_{L^{2}(\Gamma)}\right|^{2}
$$

and thus $\left\|\left(T+T^{*}\right) u\right\|^{2} \leq 2\|u\|_{L^{2}(\Gamma)}^{2}$. Second, the following relations are satisfied

$$
\begin{aligned}
\|\mathcal{T} g\|^{2}-\|g\|^{2} & =\left\|g+\mathbf{i} \omega\left(T+T^{*}\right) u\right\|^{2}-\|g\|^{2} \\
& =2 \operatorname{Re}\left(g, \mathbf{i} \omega\left(T+T^{*}\right) u\right)_{\|\cdot \cdot\|}+\omega^{2}\left\|\left(T+T^{*}\right) u\right\|^{2} \\
& =2 \operatorname{Re}(g, \mathbf{i} \omega u)_{L^{2}(\Gamma)}+\omega^{2}\left\|\left(T+T^{*}\right) u\right\|^{2} \\
& =2 \operatorname{Re}\left(\partial_{\mathbf{n}} u-\mathbf{i} \omega T u, \mathbf{i} \omega u\right)_{L^{2}(\Gamma)}+\omega^{2}\left\|\left(T+T^{*}\right) u\right\|^{2} \\
& =2 \operatorname{Re}\left(\partial_{\mathbf{n}} u, \mathbf{i} \omega u\right)_{L^{2}(\Gamma)}-2 \omega^{2} \operatorname{Re}(T u, u)_{L^{2}(\Gamma)}+\omega^{2}\left\|\left(T+T^{*}\right) u\right\|^{2} \\
& =2 \operatorname{Re}\left(\partial_{\mathbf{n}} u, \mathbf{i} \omega u\right)_{L^{2}(\Gamma)}+\omega^{2}\left(\left\|\left(T+T^{*}\right) u\right\|^{2}-\operatorname{Re}\left(\left(T+T^{*}\right) u, u\right)_{L^{2}(\Gamma)}\right) .
\end{aligned}
$$

The first term vanishes since $u$ is a solution of the homogeneous Helmholtz equation. The remaining terms cancel due to the identity above. 


\section{Two DDMs using the $2^{\text {nd }}$ order $A B C$}

This Section is dedicated to the proof that problem (16), where the boundary operator is global, can be solved using two iterative DDM algorithms. The difference between these algorithms is the level of decoupling between the subdomains. Both methods are shown to be convergent using a technique based on a suitable quadratic energy defined on the skeleton of the mesh [9]. Using a comparison argument between the iterative solution of the DDM and the exact solution, it will be shown that the quadratic energy is decreasing by a factor which controls the solution at the boundary $\Gamma$. This stability property is sufficient to show the convergence of the DDM using propagation techniques explained in $[9,3,25]$. In this work we concentrate on the essential part which concerns the stability of the quadratic energy.

We consider a decomposition of $\Omega$ into subdomains $\Omega_{i}$ for $0 \leq i \leq N_{\text {dom }}-1$ with $N_{\text {dom }} \geq 2$ the total number of subdomains. The exterior normal to a subdomain $\Omega_{i}$ is $\mathbf{n}^{i}$.

\subsection{DDM-1}

A natural DDM writes: for all subdomains, initialize $u_{i}^{0} \in H^{1}\left(\Omega_{i}\right)$ with square integrable normal derivatives and iterate for $p=0,1, \ldots$

$$
\left\{\begin{aligned}
\left(-\Delta-\omega^{2}\right) u_{i}^{p+1} & =f, & & \text { in } \Omega_{i} \\
\left(\partial_{\mathbf{n}^{i}}-\mathbf{i} \omega\right) u_{i}^{p+1} & =-\left(\partial_{\mathbf{n}^{j}}+\mathbf{i} \omega\right) u_{j}^{p}, & & \text { on } \partial \Omega_{i} \cap \partial \Omega_{j}, \forall j \neq i \\
\partial_{\mathbf{n}} u_{i}^{p+1} & =\mathbf{i} \omega T\left(u^{p+1}\right), & & \text { on } \partial \Omega_{i} \cap \Gamma .
\end{aligned}\right.
$$

The third line of equation (18) couples every $u_{i}$ together and makes this algorithm non-local. We define the following energy

$$
E^{p}:=\sum_{i=0}^{N_{\mathrm{dom}}-1} \int_{\partial \Omega_{i} \backslash \Gamma}\left|\left(\partial_{\mathbf{n}^{i}}-\mathbf{i} \omega\right) u_{i}^{p}\right|^{2} \mathrm{~d} \gamma
$$

This quantity is well defined since iterating on $p$ ensures that the normal derivatives of $u_{i}^{p}$ are square integrable provided the normal derivatives of $u_{i}^{0}$ are square integrable.

Lemma 7 The algorithm (18) is stable. For $f=0$, it has decreasing energy

$$
E^{p+1} \leq E^{p}-4 \omega^{2}\left\|u^{p}\right\|_{L^{2}(\Gamma)}^{2}
$$

Proof One has by definition

$$
E^{p+1}=\sum_{i=0}^{N_{\mathrm{dom}}-1} \int_{\partial \Omega_{i} \backslash \Gamma}\left|\left(\partial_{\mathbf{n}^{i}}-\mathbf{i} \omega\right) u_{i}^{p+1}\right|^{2} \mathrm{~d} \gamma=\sum_{j=0}^{N_{\mathrm{dom}}-1} \int_{\partial \Omega_{j} \backslash \Gamma}\left|\left(\partial_{\mathbf{n}^{j}}+\mathbf{i} \omega\right) u_{j}^{p}\right|^{2} \mathrm{~d} \gamma
$$

Algebraic manipulations and an integration by parts on the closed border $\partial \Omega_{i}$ give

$$
E^{p+1}=\sum_{j=0}^{N_{\mathrm{dom}}-1} \int_{\partial \Omega_{j} \backslash \Gamma}\left|\left(\partial_{\mathbf{n}^{j}}-\mathbf{i} \omega\right) u_{j}^{p}\right|^{2} \mathrm{~d} \gamma+4 \operatorname{Re} \sum_{j=0}^{N_{\mathrm{dom}}-1} \int_{\partial \Omega_{j} \backslash \Gamma} \frac{\partial u_{j}^{p}}{\partial \mathbf{n}^{j}} \overline{\mathbf{i} \omega u_{j}^{p}} \mathrm{~d} \gamma
$$




$$
\begin{aligned}
= & \sum_{j=0}^{N_{\mathrm{dom}}-1} \int_{\partial \Omega_{j} \backslash \Gamma}\left|\left(\partial_{\mathbf{n}^{j}}-\mathbf{i} \omega\right) u_{j}^{p}\right|^{2} \mathrm{~d} \gamma \\
& -4 \operatorname{Re} \sum_{j=0}^{N_{\mathrm{dom}}-1}\left(\int_{\partial \Omega_{j}} \mathbf{i} \omega \partial_{\mathbf{n}^{j}}\left(\frac{\left|u_{j}^{p}\right|^{2}}{2}\right) \mathrm{d} \gamma+\int_{\partial \Omega_{j} \cap \Gamma} \frac{\partial u_{j}^{p}}{\partial \mathbf{n}^{j}} \overline{\mathbf{i} \omega u_{j}^{p}} \mathrm{~d} \gamma\right) \\
= & \sum_{j=0}^{N_{\mathrm{dom}}-1} \int_{\partial \Omega_{j} \backslash \Gamma}\left|\left(\partial_{\mathbf{n}^{j}}-\mathbf{i} \omega\right) u_{j}^{p}\right|^{2} \mathrm{~d} \gamma-4 \operatorname{Re} \sum_{j=0}^{N_{\mathrm{dom}}-1} \int_{\partial \Omega_{j} \cap \Gamma} \frac{\partial u_{j}^{p}}{\partial \mathbf{n}^{j}} \overline{\mathbf{i} \omega u_{j}^{p}} \mathrm{~d} \gamma .
\end{aligned}
$$

From (15), the boundedness of $T$ and from the boundary condition on $\partial \Omega_{i} \cap \Gamma$, it follows that

$$
E^{p+1}=E^{p}-4 \omega^{2} \operatorname{Re}\left(T\left(u^{p}\right), u^{p}\right)_{L^{2}(\Gamma)} \leq E^{p}-4 \omega^{2}\left\|T\left(u^{p}\right)\right\|_{L^{2}(\Gamma)}^{2}=E^{p}-4\left\|u^{p}\right\|_{L^{2}(\Gamma)}^{2},
$$

and the claim is proven.

Remark 2 We detail here how convergence follows from this inequality. First, one has

$$
E^{p}+4 \omega^{2} \sum_{n=0}^{p-1}\left\|u^{n}\right\|_{L^{2}(\Gamma)}^{2} \leq E^{0}
$$

and this is enough to show the convergence of the trace on the boundary $\left\|u^{n}\right\|_{L^{2}(\Gamma)} \underset{n}{\rightarrow} 0$. Second, a propagation technique based on the unique continuation principle $[9,3,25]$ yields the $H^{1}$ convergence to zero in $\Omega$.

Nevertheless, this method lacks an original asset of DDMs: decoupling between local problems. Indeed, since the operator $T$ is non-local, see (13), all subdomains $\Omega_{i}$ such that $\partial \Omega_{i} \cap \Gamma \neq 0$ are coupled through the ABC.

\subsection{DDM-2: subdomains decoupled}

To restore the full-decoupling between subdomains, we start by noticing that the coupling between boundary subdomains is due to the corner conditions (11) which define the operator $T$. Since this operator is constructed through the solution of a differential form on the boundary, it is possible to decouple the subdomains using another level of DDM for the problem on the boundary.

We start by introducing the notations in line with Figure 4 . Each corner $\mathbf{A}_{k \ell}^{i j}$ is indexed by $k$ and $\ell$, as before, to know at the intersection of which edges of $\Gamma$ it corresponds, and by $i$ and $j$ to know at the intersection of which subdomains it lies. The intersection of a subdomain's border $\partial \Omega_{i}$ and of an exterior edge $\Gamma_{k}$ is noted $\Gamma_{k}^{i}:=\partial \Omega_{i} \cap \Gamma_{k}$. It can either be empty or be a segment. It is convenient to introduce the partition of nodes $\partial \Gamma_{k}^{i}=\mathcal{C}_{k}^{i} \cup \mathcal{F}_{k}^{i}$. The first set $\mathcal{C}_{k}^{i}$ contains the endpoints of $\Gamma_{k}^{i}$ that are endpoints of $\Gamma_{k}$ (and thus, corners of $\Gamma$ ), and the second set $\mathcal{F}_{k}^{i}$ contains the endpoints of $\Gamma_{k}^{i}$ that are interior points of $\Gamma_{k}$ (and thus, flat points of $\Gamma$ ). They write

$$
\mathcal{C}_{k}^{i}:=\left\{\mathbf{A}_{k \ell}^{i j}=\overline{\Gamma_{k}^{i}} \cap \overline{\Gamma_{\ell}^{j}}, \forall j, \forall \ell \neq k\right\} \quad \text { and } \quad \mathcal{F}_{k}^{i}:=\left\{\mathbf{B}_{k}^{i j}=\overline{\Gamma_{k}^{i}} \cap \overline{\Gamma_{k}^{j}}, \forall j\right\} .
$$

In the case where $\Gamma_{k}^{i}=\emptyset$, which is frequent, we set by convention $\mathcal{C}_{k}^{i}=\emptyset$ and $\mathcal{F}_{k}^{i}=\emptyset$.

An auxiliary unknown $\varphi_{i, k}$ is introduced on each segment $\Gamma_{k}^{i}$, which stands for the local value of the global quantity $T u$. Algorithm (18) is adapted as follows. For all subdomains, initialize 


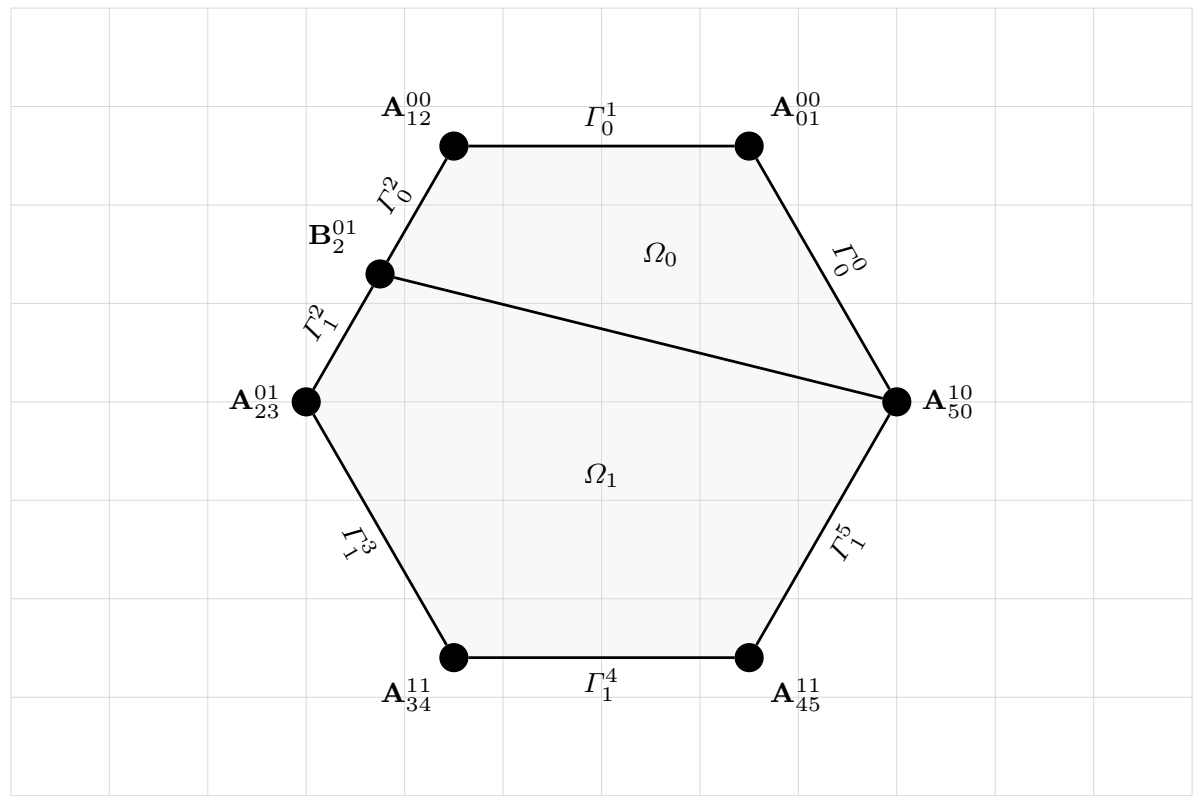

Fig. 4: Example of a hexagonal domain with two subdomains.

$u_{i}^{0} \in H^{1}\left(\Omega_{i}\right)$ with square integrable normal derivatives, and $\left(\varphi_{i, k}^{0}\right)_{k} \in \oplus_{k} H^{1}\left(\Gamma_{k}^{i}\right)$. Then, for $p=0,1, \ldots$, solve for each subdomain

$$
\begin{aligned}
& \left\{\begin{aligned}
\left(-\Delta-\omega^{2}\right) u_{i}^{p+1} & =f, & & \text { in } \Omega_{i}, \\
\left(\partial_{\mathbf{n}^{i}}-\mathbf{i} \omega\right) u_{i}^{p+1} & =-\left(\partial_{\mathbf{n}^{j}}+\mathbf{i} \omega\right) u_{j}^{p}, & & \text { on } \partial \Omega_{i} \cap \partial \Omega_{j}, \forall j \neq i, \\
\partial_{\mathbf{n}^{i}} u_{i}^{p+1} & =\mathbf{i} \omega \varphi_{i, k}^{p+1}, & & \text { on } \Gamma_{k}^{i}, \forall k,
\end{aligned}\right. \\
& \begin{cases}\left(1-\frac{1}{2 \omega^{2}} \partial_{\mathbf{t}_{k} \mathbf{t}_{k}}\right) \varphi_{i, k}^{p+1}(\mathbf{x})=u_{i}^{p+1}(\mathbf{x}), & \mathbf{x} \in \Gamma_{k}^{i}, \\
\left(\left(1+\frac{\mathbf{i} \beta}{\omega} \frac{\cos \left(\frac{\theta_{k \ell}}{2}\right)}{\cos \theta_{k \ell}}\right) \frac{\partial \varphi_{i, k}^{p+1}}{\partial \boldsymbol{\tau}_{k}}+\left(\beta-\mathbf{i} \omega \cos \left(\frac{\theta_{k \ell}}{2}\right)\right) \varphi_{i, k}^{p+1}\right)\left(\mathbf{A}_{k \ell}^{i j}\right) & \\
=\left(\left(-1+\frac{\mathbf{i} \beta}{\omega} \frac{\cos \left(\frac{\theta_{k \ell}}{2}\right)}{\cos \theta_{k \ell}}\right) \frac{\partial \varphi_{j, \ell}^{p}}{\partial \boldsymbol{\tau}_{\ell}}+\left(\beta+\mathbf{i} \omega \cos \left(\frac{\theta_{k \ell}}{2}\right)\right) \varphi_{j, \ell}^{p}\right)\left(\mathbf{A}_{k \ell}^{i j}\right), & \forall \mathbf{A}_{k \ell}^{i j} \in \mathcal{C}_{k}^{i}, \\
\left(\partial_{\boldsymbol{\tau}_{k}}+\mathbf{i} \beta\right) \varphi_{i, k}^{p+1}\left(\mathbf{B}_{k}^{i j}\right)=\left(-\partial_{\boldsymbol{\tau}_{k}}+\mathbf{i} \beta\right) \varphi_{j, k}^{p}\left(\mathbf{B}_{k}^{i j}\right), & \forall \mathbf{B}_{k}^{i j} \in \mathcal{F}_{k}^{i} .\end{cases}
\end{aligned}
$$

Remark 3 As stated in Section 2, the singularity $\frac{\beta}{\cos \left(\theta_{k \ell}\right)}$ for $\theta_{k \ell}=-\frac{\pi}{2},-\frac{3 \pi}{2}$ is artificial. It is systematically removed by taking $\beta=-|\omega| \cos \left(\theta_{k \ell}\right)$, see (10).

The resolution of (19)-(20) can be done in parallel for all subdomains by solving local problems in $\Omega_{i} \times\left(\oplus_{k} \Gamma_{k}^{i}\right)$ with unknowns $\left(u_{i}^{p+1},\left(\varphi_{i, k}^{p+1}\right)_{k}\right)$. Once again, we prove the algorithm is endowed 
to a decreasing energy for $f=0$. This energy is defined by

$$
\begin{aligned}
F^{p}:= & \sum_{i=0}^{N_{\mathrm{dom}}-1}\left(\int_{\partial \Omega_{i} \backslash \Gamma}\left|\left(\partial_{\mathbf{n}^{i}}-\mathbf{i} \omega\right) u_{i}^{p}\right|^{2} \mathrm{~d} \gamma\right. \\
& +\sum_{\substack{k=0 \\
\mathbf{A}_{k \ell}^{i j} \in \mathcal{C}_{k}^{i}}}^{K-1} \frac{1}{2 \beta\left(1-\frac{\cos ^{2}\left(\frac{\theta_{k \ell}}{2}\right)}{\cos \theta_{k \ell}}\right)}\left|\left(1+\frac{\mathbf{i} \beta}{\omega} \frac{\cos \left(\frac{\theta_{k \ell}}{2}\right)}{\cos \theta_{k \ell}}\right) \frac{\partial \varphi_{i, k}^{p}}{\partial \boldsymbol{\tau}_{k}}+\left(\beta-\mathbf{i} \omega \cos \left(\frac{\theta_{k \ell}}{2}\right)\right) \varphi_{i, k}^{p}\right|^{2}\left(\mathbf{A}_{k \ell}^{i j}\right) \\
& \left.+\sum_{\substack{k=0 \\
\mathbf{B}_{k}^{i j} \in \mathcal{F}_{k}^{i}}}^{K-1} \frac{1}{2 \omega}\left|\partial_{\boldsymbol{\tau}_{k}} \varphi_{i, k}^{p}+\omega \varphi_{i, k}^{p}\right|^{2}\left(\mathbf{B}_{k}^{i j}\right)\right) .
\end{aligned}
$$

Remark 4 We explain here the choice for the sign of $\beta$ : it is determined by the non negativity of energy $F^{p}$. For $\beta=b \omega \cos \theta_{k \ell}$, the denominator on the second line of the definition of $F^{p}$ rewrites

$$
2 \beta\left(1-\frac{\cos ^{2}\left(\frac{\theta_{k \ell}}{2}\right)}{\cos \theta_{k \ell}}\right)=2 b \omega\left(\cos ^{2}\left(\frac{\theta_{k \ell}}{2}\right)-1\right)=-2 b \omega \sin ^{2}\left(\frac{\theta_{k \ell}}{2}\right),
$$

and is positive for $b \operatorname{sign} \omega<0$. Therefore, we take $b:=-\operatorname{sign} \omega$ and it justifies the choice $\beta=-|\omega| \cos \theta_{k \ell}$ introduced previously.

Lemma 8 The algorithm (19)-(20) is stable. For $f=0$, it has decreasing energy

$$
F^{p+1}=F^{p}-2 \sum_{j=0}^{N_{d o m}-1} \sum_{\ell=0}^{K-1} \int_{\Gamma_{\ell}^{j}}\left(2 \omega^{2}\left|\varphi_{j, \ell}^{p}\right|^{2}+\left|\partial_{\mathbf{t}_{\ell}} \varphi_{j, \ell}^{p}\right|^{2}\right) \mathrm{d} \gamma .
$$

Proof Using the definition of the energy $F^{p+1}$ and of the quantities $u_{i}^{p+1}$ and $\varphi_{i, k}^{p+1}$, it gives

$$
\begin{aligned}
& F^{p+1}=\sum_{i=0}^{N_{\text {dom }}-1}\left(\int_{\partial \Omega_{i} \backslash \Gamma}\left|\left(\partial_{\mathbf{n}^{i}}-\mathbf{i} \omega\right) u_{i}^{p+1}\right|^{2} \mathrm{~d} \gamma+\sum_{\substack{k=0 \\
\mathbf{B}_{k}^{i j} \in \mathcal{F}_{k}^{i}}}^{K-1} \frac{1}{2 \omega}\left|\omega \varphi_{i, k}^{p+1}+\partial_{\boldsymbol{\tau}_{k}} \varphi_{i, k}^{p+1}\right|^{2}\left(\mathbf{B}_{k}^{i j}\right)\right. \\
& \left.+\sum_{\substack{k=0 \\
\mathbf{A}_{k \ell}^{i j} \in \mathcal{C}_{k}^{i}}}^{K-1} \frac{1}{2|\omega| \sin ^{2}\left(\frac{\theta_{k \ell}}{2}\right)}\left|\left(1+\frac{\mathbf{i} \beta}{\omega} \frac{\cos \left(\frac{\theta_{k \ell}}{2}\right)}{\cos \theta_{k \ell}}\right) \frac{\partial \varphi_{i, k}^{p+1}}{\partial \boldsymbol{\tau}_{k}}+\left(\beta-\mathbf{i} \omega \cos \left(\frac{\theta_{k \ell}}{2}\right)\right) \varphi_{i, k}^{p+1}\right|^{2}\left(\mathbf{A}_{k \ell}^{i j}\right)\right) \\
& =\sum_{j=0}^{N_{\mathrm{dom}}-1}\left(\int_{\partial \Omega_{j} \backslash \Gamma}\left|\left(\partial_{\mathbf{n}^{j}}+\mathbf{i} \omega\right) u_{j}^{p}\right|^{2} \mathrm{~d} \gamma+\sum_{\substack{\ell=0 \\
\mathbf{B}_{\ell}^{i j} \in \mathcal{F}_{\ell}^{j}}}^{K-1} \frac{1}{2 \omega}\left|\omega \varphi_{j, \ell}^{p}-\partial_{\boldsymbol{\tau}_{\ell}} \varphi_{j, \ell}^{p}\right|^{2}\left(\mathbf{B}_{\ell}^{i j}\right)\right. \\
& \left.+\sum_{\substack{\ell=0 \\
\mathbf{A}_{k \ell}^{i j} \in \mathcal{C}_{\ell}^{j}}}^{K-1} \frac{1}{2|\omega| \sin ^{2}\left(\frac{\theta_{k \ell}}{2}\right)}\left|\left(-1+\frac{\mathbf{i} \beta}{\omega} \frac{\cos \left(\frac{\theta_{k \ell}}{2}\right)}{\cos \theta_{k \ell}}\right) \frac{\partial \varphi_{j, \ell}^{p}}{\partial \boldsymbol{\tau}_{\ell}}+\left(\beta+\mathbf{i} \omega \cos \left(\frac{\theta_{k \ell}}{2}\right)\right) \varphi_{j, \ell}^{p}\right|^{2}\left(\mathbf{A}_{k \ell}^{i j}\right)\right)
\end{aligned}
$$


The integrals on the boundary are treated as in the proof of Lemma 7. One has

$$
\sum_{j=0}^{N_{\mathrm{dom}}-1} \int_{\partial \Omega_{j} \backslash \Gamma}\left|\left(\partial_{\mathbf{n}^{j}}+\mathbf{i} \omega\right) u_{j}^{p}\right|^{2} \mathrm{~d} \gamma=\sum_{j=0}^{N_{\mathrm{dom}}-1} \int_{\partial \Omega_{j} \backslash \Gamma}\left|\left(\partial_{\mathbf{n}^{j}}-\mathbf{i} \omega\right) u_{j}^{p}\right|^{2} \mathrm{~d} \gamma-4 \sum_{j=0}^{N_{\mathrm{dom}}-1} \operatorname{Re} \int_{\partial \Omega_{j} \cap \Gamma} \frac{\partial u_{j}^{p}}{\partial \mathbf{n}^{j}} \overline{\mathbf{i} \omega u_{j}^{p}} .
$$

For the terms concerning the non flat corners $\mathbf{A}_{k \ell}^{i j}$, similar algebraic relations yield

$$
\begin{aligned}
& \sum_{\substack{\ell=0 \\
\mathbf{A}_{k \ell}^{i j} \in \mathcal{C}_{\ell}^{j}}}^{K-1} \frac{1}{2|\omega| \sin ^{2}\left(\frac{\theta_{k \ell}}{2}\right)}\left|\left(-1+\frac{\mathbf{i} \beta}{\omega} \frac{\cos \left(\frac{\theta_{k \ell}}{2}\right)}{\cos \theta_{k \ell}}\right) \frac{\partial \varphi_{j, \ell}^{p}}{\partial \boldsymbol{\tau}_{\ell}}+\left(\beta+\mathbf{i} \omega \cos \left(\frac{\theta_{k \ell}}{2}\right)\right) \varphi_{j, \ell}^{p}\right|^{2}\left(\mathbf{A}_{k \ell}^{i j}\right) \\
& =\sum_{\substack{\ell=0 \\
\mathbf{A}_{k \ell}^{i j} \in \mathcal{C}_{\ell}^{j}}}^{K-1} \frac{1}{2|\omega| \sin ^{2}\left(\frac{\theta_{k \ell}}{2}\right)}\left|\left(1+\frac{\mathbf{i} \beta}{\omega} \frac{\cos \left(\frac{\theta_{k \ell}}{2}\right)}{\cos \theta_{k \ell}}\right) \frac{\partial \varphi_{j, \ell}^{p}}{\partial \boldsymbol{\tau}_{\ell}}+\left(\beta-\mathbf{i} \omega \cos \left(\frac{\theta_{k \ell}}{2}\right)\right) \varphi_{j, \ell}^{p}\right|^{2}\left(\mathbf{A}_{k \ell}^{i j}\right) \\
& -4 \operatorname{Re} \sum_{\substack{\ell=0 \\
\mathbf{A}_{k \ell}^{i j} \in \mathcal{C}_{\ell}^{j}}}^{K-1} \frac{1}{2|\omega| \sin ^{2}\left(\frac{\theta_{k \ell}}{2}\right)}\left(\frac{\mathbf{i} \beta}{\omega} \frac{\cos \left(\frac{\theta_{k \ell}}{2}\right)}{\cos \theta_{k \ell}} \frac{\partial \varphi_{j, \ell}^{p}}{\partial \boldsymbol{\tau}_{\ell}}+\beta \varphi_{j, \ell}^{p}\right) \overline{\left(\frac{\partial \varphi_{j, \ell}^{p}}{\partial \boldsymbol{\tau}_{\ell}}-\mathbf{i} \omega \cos \left(\frac{\theta_{k \ell}}{2}\right) \varphi_{j, \ell}^{p}\right)}\left(\mathbf{A}_{k \ell}^{i j}\right) \\
& =\sum_{\substack{\ell=0 \\
\mathbf{A}_{k \ell}^{i j} \in \mathcal{C}_{\ell}^{j}}}^{K-1} \frac{1}{2|\omega| \sin ^{2}\left(\frac{\theta_{k \ell}}{2}\right)}\left|\left(1+\frac{\mathbf{i} \beta}{\omega} \frac{\cos \left(\frac{\theta_{k \ell}}{2}\right)}{\cos \theta_{k \ell}}\right) \frac{\partial \varphi_{j, \ell}^{p}}{\partial \boldsymbol{\tau}_{\ell}}+\left(\beta-\mathbf{i} \omega \cos \left(\frac{\theta_{k \ell}}{2}\right)\right) \varphi_{j, \ell}^{p}\right|^{2}\left(\mathbf{A}_{k \ell}^{i j}\right) \\
& -4 \operatorname{Re} \sum_{\substack{\ell=0 \\
\mathbf{A}_{k \ell}^{i j} \in \mathcal{C}_{\ell}^{j}}}^{K-1} \frac{1}{2|\omega| \sin ^{2}\left(\frac{\theta_{k \ell}}{2}\right)}\left(\mathbf{i} b \cos \left(\frac{\theta_{k \ell}}{2}\right) \frac{\partial \varphi_{j, \ell}^{p}}{\partial \boldsymbol{\tau}_{\ell}}+b \omega \cos \theta_{k \ell} \varphi_{j, \ell}^{p}\right) \overline{\left(\frac{\partial \varphi_{j, \ell}^{p}}{\partial \boldsymbol{\tau}_{\ell}}-\mathbf{i} \omega \cos \left(\frac{\theta_{k \ell}}{2}\right) \varphi_{j, \ell}^{p}\right)}\left(\mathbf{A}_{k \ell}^{i j}\right) \\
& =\sum_{\substack{\ell=0 \\
\mathbf{A}_{k \ell}^{i j} \in \mathcal{C}_{\ell}^{j}}}^{K-1} \frac{1}{2|\omega| \sin ^{2}\left(\frac{\theta_{k \ell}}{2}\right)}\left|\left(1+\frac{\mathbf{i} \beta}{\omega} \frac{\cos \left(\frac{\theta_{k \ell}}{2}\right)}{\cos \theta_{k \ell}}\right) \frac{\partial \varphi_{j, \ell}^{p}}{\partial \boldsymbol{\tau}_{\ell}}+\left(\beta-\mathbf{i} \omega \cos \left(\frac{\theta_{k \ell}}{2}\right)\right) \varphi_{j, \ell}^{p}\right|^{2}\left(\mathbf{A}_{k \ell}^{i j}\right) \\
& -2 \operatorname{Re} \sum_{\substack{\ell=0 \\
\mathbf{A}_{k \ell}^{i j} \in \mathcal{C}_{\ell}^{j}}}^{K-1} \frac{\partial \varphi_{j, \ell}^{p}}{\partial \boldsymbol{\tau}_{\ell}}\left(\mathbf{A}_{k \ell}^{i j}\right) \overline{\varphi_{j, \ell}^{p}}\left(\mathbf{A}_{k \ell}^{i j}\right)
\end{aligned}
$$

Similarly, for the flat corners $\mathbf{B}_{\ell}^{i j}$, one has

$$
\begin{aligned}
\sum_{\substack{\ell=0 \\
\mathbf{B}_{\ell}^{i j} \in \mathcal{F}_{\ell}^{j}}}^{K-1} \frac{1}{2 \omega}\left|\omega \varphi_{j, \ell}^{p}-\partial_{\boldsymbol{\tau}_{\ell}} \varphi_{j, \ell}^{p}\right|^{2}\left(\mathbf{B}_{\ell}^{i j}\right)= & \sum_{\substack{\ell=0 \\
\mathbf{B}_{\ell}^{i j} \in \mathcal{F}_{\ell}^{j}}}^{K-1} \frac{1}{2 \omega}\left|\omega \varphi_{j, \ell}^{p}+\partial_{\boldsymbol{\tau}_{\ell}} \varphi_{j, \ell}^{p}\right|^{2}\left(\mathbf{B}_{\ell}^{i j}\right) \\
& -2 \operatorname{Re} \sum_{\substack{\ell=0 \\
\mathbf{B}_{\ell}^{i j} \in \mathcal{F}_{\ell}^{j}}}^{K-1} \frac{\partial \varphi_{j, \ell}^{p}}{\partial \boldsymbol{\tau}_{\ell}}\left(\mathbf{B}_{\ell}^{i j}\right) \overline{\varphi_{j, \ell}^{p}}\left(\mathbf{B}_{\ell}^{i j}\right) .
\end{aligned}
$$

Therefore, it holds that

$$
F^{p+1}=F^{p}-4 \operatorname{Re} \sum_{j=0}^{N_{\mathrm{dom}}-1} \int_{\partial \Omega_{j} \cap \Gamma} \frac{\partial u_{j}^{p}}{\partial \mathbf{n}^{j}} \overline{\mathbf{i} \omega u_{j}^{p}} \mathrm{~d} \gamma-2 \operatorname{Re} \sum_{j=0}^{N_{\mathrm{dom}}-1} \sum_{\substack{\ell \\ \mathbf{A} \in \mathcal{C}_{\ell}^{j} \cup \mathcal{F}_{\ell}^{j}}} \frac{\partial \varphi_{j, \ell}^{p}}{\partial \boldsymbol{\tau}_{\ell}}(\mathbf{A}) \overline{\varphi_{j, \ell}^{p}}(\mathbf{A}) .
$$


It remains to manipulate the two last terms. It follows from (19) that for all $j$

$$
\int_{\partial \Omega_{j} \cap \Gamma} \frac{\partial u_{j}^{p}}{\partial \mathbf{n}^{j}} \overline{\mathbf{i} \omega u_{j}^{p}} \mathrm{~d} \gamma=\sum_{\ell=0}^{K-1} \int_{\Gamma_{\ell}^{j}} \frac{\partial u_{j}^{p}}{\partial \mathbf{n}^{j}} \overline{\mathbf{i} \omega u_{j}^{p}} \mathrm{~d} \gamma=\sum_{\ell=0}^{K-1} \int_{\Gamma_{\ell}^{j}} \omega^{2} \varphi_{j, \ell}^{p} \overline{u_{j}^{p}} \mathrm{~d} \gamma .
$$

Integrating by parts the equation from $(20)$ at iteration $p$ on $\Gamma_{\ell}^{j}$ against $\varphi_{j, \ell}^{p}$ and taking the sum over all subdomain and edge indices $j$ and $\ell$ yields

$$
\begin{array}{r}
\sum_{j=0}^{N_{\text {dom }}} \sum_{\ell=0}^{-1 K-1}\left(\int_{\Gamma_{\ell}^{j}}\left(\left|\varphi_{j, \ell}^{p}\right|^{2}+\frac{1}{2 \omega^{2}}\left|\frac{\partial \varphi_{j, \ell}^{p}}{\partial \mathbf{t}_{\ell}}\right|^{2}\right) \mathrm{d} \gamma-\frac{1}{2 \omega^{2}} \sum_{\mathbf{A} \in \mathcal{C}_{\ell}^{j} \cup \mathcal{F}_{\ell}^{j}} \frac{\partial \varphi_{j, \ell}^{p}}{\partial \boldsymbol{\tau}_{\ell}}(\mathbf{A}) \overline{\varphi_{j, \ell}^{p}}(\mathbf{A})\right) \\
=\sum_{j=0}^{N_{\mathrm{dom}}-1 K-1} \sum_{\ell=0}^{K} \int_{\Gamma_{\ell}^{j}} u_{j}^{p} \overline{\varphi_{j, \ell}^{p}} \mathrm{~d} \gamma
\end{array}
$$

Therefore $F^{p+1}=F^{p}-4 \sum_{j=0}^{N_{\mathrm{dom}}-1} \sum_{\ell=0}^{K-1} \int_{\Gamma_{\ell}^{j}}\left(\omega^{2}\left|\varphi_{j, \ell}^{p}\right|^{2}+\frac{1}{2}\left|\frac{\partial \varphi_{j, \ell}^{p}}{\partial \mathbf{t}_{\ell}}\right|^{2}\right) \mathrm{d} \gamma$ and the proof is ended.

Algorithm (19)-(20) has the advantage of restoring a full-decoupling with respect to the subdomains. There remains some coupling between $u_{i}^{p+1}$ and $\left(\varphi_{i, k}^{p+1}\right)_{k}$ since in the DDM defining $u_{i}^{p+1}$, it is imposed that $\partial_{\mathbf{n}^{i}} u_{i}^{p+1}=\mathbf{i} \omega \varphi_{i, k}^{p+1}$ on each $\Gamma_{k}^{i}$, and that at the same time, in the DDM defining $\varphi_{i, k}^{p+1}$, it is imposed that $\varphi_{i, k}^{p+1}-\partial_{\mathbf{t}_{k} \mathbf{t}_{k}} \varphi_{i, k}^{p+1} /\left(2 \omega^{2}\right)=u_{i}^{p+1}$ on $\Gamma_{k}^{i}$. Decoupling the systems (19) and (20) is possible using another level of DDM, and allows to solve the equation on $u_{i}^{p+1}$ as a classical Helmholtz boundary value problem. This new method introduces new unknowns and complicates the expression of the energy, but does not require any new mathematical concept, see DDM-3 in Appendix B.

\section{A DDM with transmission conditions for corners}

Based on the decreasing energy endowed ABC derived previously, our goal is now to generalize the corner approach to the definition of transmission conditions for subdomains with broken line interfaces. This situation is often encountered in applications, when using a mesh generator for example. A first difference is that a $2^{\text {nd }}$ order $\mathrm{ABC}$ is a model for radiation condition while a $2^{\text {nd }}$ order TC is essentially a preconditioning of the continuity conditions

$$
u_{i}-u_{j}=0 \quad \text { and } \quad \frac{\partial u_{i}}{\partial \mathbf{n}^{i}}+\frac{\partial u_{j}}{\partial \mathbf{n}^{j}}=0 \quad \text { on } \partial \Omega_{i} \cap \partial \Omega_{j} .
$$

A second difference is that the reformulation of (21) must be localized on interfaces to be compatible with the notion of DDM.

To do so, we define local operators $R_{i j}$ and $R_{j i}$ defined on $\partial \Omega_{i} \cap \partial \Omega_{j}$ and we replace the continuity relations (21) by

$$
\left\{\begin{array}{l}
\left(\partial_{\mathbf{n}^{i}}-\mathbf{i} \omega R_{i j}\right) u_{i}=-\left(\partial_{\mathbf{n}^{i}}+\mathbf{i} \omega R_{i j}\right) u_{j} \\
\left(\partial_{\mathbf{n}^{j}}-\mathbf{i} \omega R_{j i}\right) u_{j}=-\left(\partial_{\mathbf{n}^{j}}+\mathbf{i} \omega R_{j i}\right) u_{i}
\end{array}\right.
$$

To construct operators $R_{i j}$ and $R_{j i}$, we start from the same second order differential relation as for the $\mathrm{ABC}$

$$
\left(1-\frac{1}{2 \omega^{2}} \partial_{\mathbf{t t}}\right) \partial_{\mathbf{n}} u=\mathbf{i} \omega u
$$


and incorporate different features, as boundary conditions for the operator $\partial_{\mathbf{t t}}$, so as to construct $R_{i j} \approx\left(1-\frac{1}{2 \omega^{2}} \partial_{\mathbf{t t}}\right)^{-1}$. An originality of our construction with respect to the literature $[25,28$, $30]$ is that $R_{i j} \neq R_{j i}$ if the angle at the corner is non trivial. However we will see that $R_{j i}=R_{i j}^{*}$ by construction and using the new isometry Lemma 6 , it is the cornerstone of the mathematical justification of this construction. We recall that we adopt in this work the distinction between corners and cross-points which is common in the literature (see for example [26]). Whenever an endpoint of a segment lies on the interface of two subdomains only or is an angular point of the exterior boundary, we call it a corner. All the other endpoints are called cross-points, and lie on the interfaces of at least three subdomains. We remind the reader that we only consider in this work subdomain decompositions that do not contain cross-points.

\subsection{Description of the interfaces}

We consider polygonal subdomains $\Omega_{i}$ for $i=0, \ldots, N_{\mathrm{dom}}-1$, and introduce the notations corresponding to Figure 5. Two adjacent subdomains $\Omega_{i}$ and $\Omega_{j}$ share a broken-line interface $\Delta^{i j}=\Delta^{j i}=\partial \Omega_{i} \cap \partial \Omega_{j}$ in common. The broken line $\Delta^{i j}$ is composed of $m_{i j}$ segments $\Delta^{i j}=$ $\cup_{r=0}^{m_{i j}-1} \Delta_{r}^{i j}$, for $m_{i j} \geq 1$. If $m_{i j}=1$, the interface is a straight line, and the extremities lie on the global boundary $\Gamma$. Otherwise, the interface is a broken line, and in addition to the extremities, there are corner points that belong to $\Delta^{i j}$. In that second case, the segments $\Delta_{r}^{i j}$ are numbered consecutively in the counter-clockwise order according to $\Omega_{i}$. Inverting the roles of $i$ and $j$, the relation $\Delta_{r}^{j i}=\Delta_{m_{i j}-1-r}^{i j}$ thus holds. As in the previous section, it is convenient to introduce a partition of nodes $\partial \Delta_{r}^{i j}=\mathcal{C}_{r}^{i j} \cup \mathcal{N}_{r}^{i j}$. Indeed, since cross-point are discarded from our configuration, a segment $\Delta_{r}^{i j}$ can have two types of endpoints: they are either corner points of $\Delta^{i j}$ and belong to $\overline{\Delta_{r}^{i j}} \cap \overline{\Delta_{s}^{i j}}$ for given $r$ and $s$, or lie on the boundary $\Gamma$, The first category forms the set $\mathcal{C}_{r}^{i j}$ and the second category forms the set $\mathcal{N}_{r}^{i j}$ :

$$
\mathcal{C}_{r}^{i j}:=\left\{\mathbf{Q}_{r s}^{i j}=\overline{\Delta_{r}^{i j}} \cap \overline{\Delta_{s}^{i j}}, \forall s \neq r\right\}, \quad \mathcal{N}_{r}^{i j}:=\left\{\mathbf{P}=\overline{\Delta_{r}^{i j}} \cap \Gamma\right\} .
$$

At a corner $\mathbf{Q}_{r s}^{i j}$, we define $\theta_{r s}^{i j} \in(-2 \pi, 2 \pi) \backslash\{0\}$ the angle from $\Delta_{r}^{i j}$ to $\Delta_{s}^{i j}$. The degenerate cases $\theta_{r s}^{i j}= \pm \pi$ are set aside as they correspond to flat corners. Exchanging the roles of $i$ and $j$, the following relation holds:

$$
\theta_{r s}^{i j}=-2 \pi-\theta_{m_{i j}-1-s, m_{i j}-1-r}^{j i} \in(-2 \pi, 2 \pi) \backslash\{-\pi, 0, \pi\} .
$$

Once again, on the flat parts of $\Delta^{i j}, \mathbf{t}^{i}$ is the counter-clockwise oriented tangent vector to $\Omega_{i}$ and $\mathbf{n}^{i}=\left(\mathbf{t}^{i}\right)^{\perp}$ is the outgoing normal vector. By construction, one has $\mathbf{t}^{i}=-\mathbf{t}^{j}$ and $\mathbf{n}^{i}=-\mathbf{n}^{j}$. At the endpoints of a segment $\Delta_{r}^{i j}, \boldsymbol{\tau}_{r}^{i}$ is the tangential vector pointing outwards, see Figure 2.

\subsection{Adapting the corner strategy for ABC to define a TC}

The design principle of the new TC is to adopt a form that writes with a new operator $R_{i j}$

$$
\frac{\partial u_{i}^{p+1}}{\partial \mathbf{n}^{i}}-\mathbf{i} \omega R_{i j} u_{i}^{p+1}=-\frac{\partial u_{j}^{p}}{\partial \mathbf{n}^{j}}-\mathbf{i} \omega R_{i j} u_{j}^{p}, \quad \text { on } \Delta^{i j} .
$$

Many operators fall into this setting [25]. The original idea here is to mimic the $2^{\text {nd }}$ order cornerbased $\mathrm{ABC}$ from (18) to treat the $\mathcal{C}_{r}^{i j}$ corners. We will use homogeneous Neumann boundary 


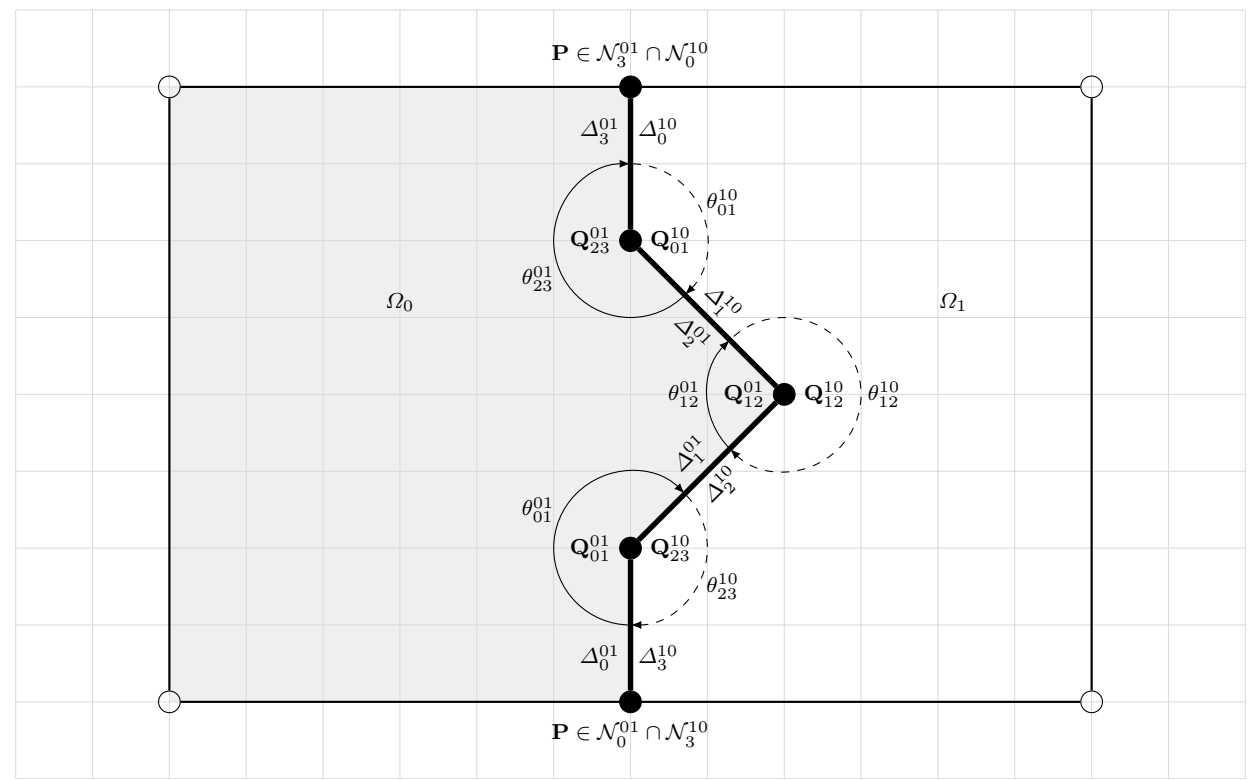

Fig. 5: Example of a $N_{\text {dom }}=2$ subdomain decomposition with a $m_{i j}=4$ segments broken-line interface.

conditions for the $\mathcal{N}_{r}^{i j}$ nodes. For an unknown $\varphi_{i, r}$ defined on $\Delta_{r}^{i j}$ for $0 \leq r \leq m_{i j}-1$ and fixed $0 \leq i \leq N_{\text {dom }}-1$, we adapt relations (8) to get

$$
\begin{cases}\partial_{\boldsymbol{\tau}_{r}^{i}} \varphi_{i, r}\left(\mathbf{Q}_{r s}^{i j}\right)-\frac{\mathbf{i} \omega}{2}\left(\frac{\cos \left(\theta_{r s}^{i j}\right)}{\cos \left(\frac{\theta_{r s}^{i j}}{2}\right)}+\cos \left(\frac{\theta_{r s}^{i j}}{2}\right)\right) \varphi_{i, r}\left(\mathbf{Q}_{r s}^{i j}\right) & \\ =\frac{\mathbf{i} \omega}{2}\left(-\frac{\cos \left(\theta_{r s}^{i j}\right)}{\cos \left(\frac{\theta_{r s}^{i j}}{2}\right)}+\cos \left(\frac{\theta_{r s}^{i j}}{2}\right)\right) \varphi_{i, s}\left(\mathbf{Q}_{r s}^{i j}\right), & \forall \mathbf{Q}_{r s}^{i j} \in \mathcal{C}_{r}^{i j}, \\ \partial_{\boldsymbol{\tau}_{r}^{i}}(\mathbf{P})=0, & \forall \mathbf{P} \in \mathcal{N}_{r}^{i j} .\end{cases}
$$

The quantities are well defined as $\cos \left(\frac{\theta_{r s}^{i j}}{2}\right) \neq 0$ since $\theta_{r s}^{i j} \neq \pm \pi$. The above system yields $2\left(m_{i j}-2\right)$ linear relations for $\cup_{r} \mathcal{C}_{r}^{i j}$, which we will use below.

Remark 5 Relation (22) implies that $\cos \left(\frac{\theta_{r s}^{i j}}{2}\right)=-\cos \left(\frac{\theta_{m_{i j}-1-s, m_{i j}-1-r}^{j i}}{2}\right)$ and $\cos \left(\theta_{r s}^{i j}\right)=$ $\cos \left(\theta_{m_{i j}-1-s, m_{i j}-1-r}^{j i}\right)$. Therefore, writing (24) for $\Omega_{j}$ instead of $\Omega_{i}$ comes down to taking the conjugate of each coefficient.

We then define operator $R_{i j}$, which replicates the variational formulation (13), but in a different geometric setting and with a different functional space. Instead of $\Gamma$ and $\oplus_{k} H^{1}\left(\Gamma_{k}\right)$ for $k=0, \ldots, K-1$, we consider $\Delta^{i j}$ and the space $\oplus_{r} H^{1}\left(\Delta_{r}^{i j}\right)$ for $r=0, \ldots, m_{i j}-1$. 
Definition 2 Let $R_{i j}: L^{2}\left(\Delta^{i j}\right) \rightarrow L^{2}\left(\Delta^{i j}\right)$ be the boundary operator such that, for $u \in L^{2}\left(\Delta^{i j}\right)$, $R_{i j}(u)=\varphi \in \oplus_{r} H^{1}\left(\Delta_{r}^{i j}\right)$, where $\varphi_{r}=\left.\varphi\right|_{\Delta_{r}^{i j}}$, is the unique variational solution of

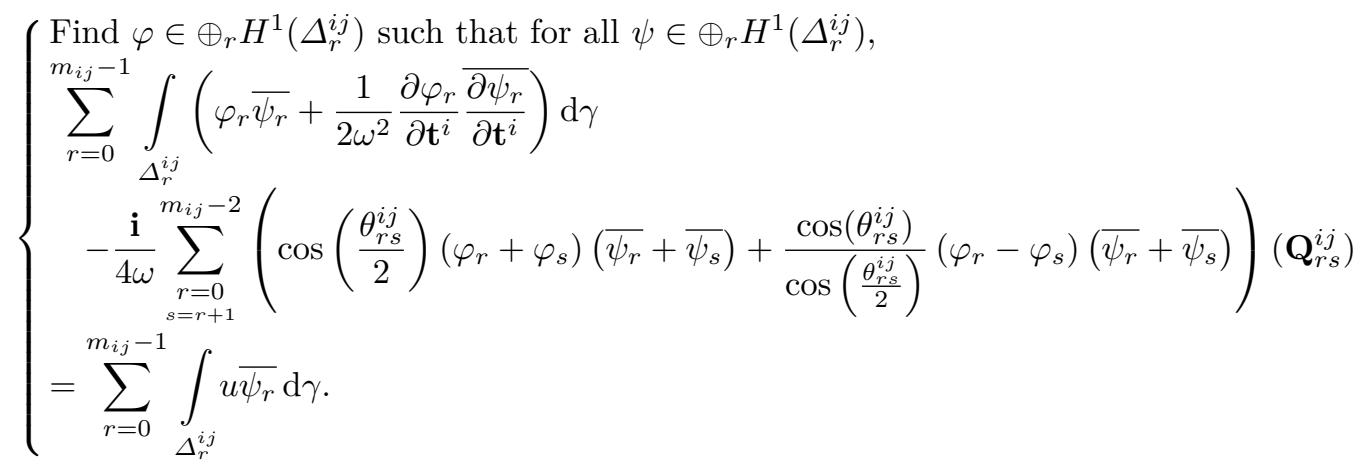

We now write the DDM algorithm. Initialize $u_{i}^{0} \in H^{1}\left(\Omega_{i}\right)$ with square integrable derivatives in each subdomain. Then, for $p=0,1, \ldots$, solve for each subdomain

$$
\left\{\begin{aligned}
\left(-\Delta-\omega^{2}\right) u_{i}^{p+1} & =f, & & \text { in } \Omega_{i}, \\
\frac{\partial u_{i}^{p+1}}{\partial \mathbf{n}^{i}}-\mathbf{i} \omega R_{i j}\left(u_{i}^{p+1}\right) & =-\frac{\partial u_{j}^{p}}{\partial \mathbf{n}^{j}}-\mathbf{i} \omega R_{i j}\left(u_{j}^{p}\right) & & \text { on } \Delta^{i j}, \forall j \neq i, \\
\left(\partial_{\mathbf{n}^{i}}-\mathbf{i} \omega\right) u_{i}^{p+1} & =0, & & \text { on } \partial \Omega_{i} \cap \Gamma .
\end{aligned}\right.
$$

The following lemma states that the operator of the interface has similar properties to the one of the global boundary problem studied in Section 2.4.

Lemma 9 The family of operators $\left(R_{i j}\right)_{i, j}$ satisfies $R_{j i}=R_{i j}^{*}$, and $R_{i j}+R_{j i}$ is positive.

Proof This a direct consequence of Remark 5: inverting the role of $i$ and $j$ in $R_{i j}$ changes the sign of the corner quantities in the bilinear form, see the second line of the expression in Definition 2. The positivity is obtained using the same arguments as in the proof of Lemma 5.

We once more prove that the algorithm is endowed to a decreasing energy for $f=0$. Define the energy

$$
J^{p}:=2 \sum_{i} \sum_{j}\left\|\left(R_{i j}+R_{j i}\right)^{-1 / 2}\left(\frac{\partial u_{i}^{p}}{\partial \mathbf{n}^{i}}-\mathbf{i} \omega R_{i j} u_{i}^{p}\right)\right\|_{L^{2}\left(\partial \Omega_{i} \cap \partial \Omega_{j}\right)}^{2} .
$$

Under convenient integrability assumptions, it can be rewritten as

$$
J^{p}=2 \sum_{i} \sum_{j} \int_{\partial \Omega_{i} \cap \partial \Omega_{j}}\left(R_{i j}+R_{j i}\right)^{-1}\left(\frac{\partial u_{i}^{p}}{\partial \mathbf{n}^{i}}-\mathbf{i} \omega R_{i j} u_{i}^{p}\right)\left(\overline{\frac{\partial u_{i}^{p}}{\partial \mathbf{n}^{i}}-\mathbf{i} \omega R_{i j} u_{i}^{p}}\right) \mathrm{d} \gamma .
$$

Lemma 10 The algorithm (25) is stable. For $f=0$, it has decreasing energy

$$
J^{p+1}=J^{p}-4 \omega^{2} \sum_{i} \int_{\partial \Omega_{i} \cap \Gamma}\left|u_{i}^{p}\right|^{2} \mathrm{~d} \gamma .
$$

Proof Using the transmission condition, one has

$$
J^{p+1}=2 \sum_{i} \sum_{j} \int_{\partial \Omega_{i} \cap \partial \Omega_{j}}\left(R_{i j}+R_{j i}\right)^{-1}\left(\frac{\partial u_{j}^{p}}{\partial \mathbf{n}^{j}}+\mathbf{i} \omega R_{i j} u_{j}^{p}\right)\left(\overline{\frac{\partial u_{j}^{p}}{\partial \mathbf{n}^{j}}+\mathbf{i} \omega R_{i j} u_{j}^{p}}\right) \mathrm{d} \gamma
$$


Define $R_{i i}$ as the identity operator on $\partial \Omega_{i} \cap \Gamma$. Decompose $v \in L^{2}\left(\partial \Omega_{i}\right)=L^{2}\left(\partial \Omega_{i} \cap \Gamma\right) \oplus_{j \neq i}$ $L^{2}\left(\partial \Omega_{i} \cap \partial \Omega_{j}\right)$ as $v_{i i}=\left.v\right|_{\partial \Omega_{i} \cap \Gamma}$ and $v_{i j}=\left.v\right|_{\partial \Omega_{i} \cap \partial \Omega_{j}}$. Each subdomain can thus be interpreted as an individual domain, associated to an operator defined on its exterior boundary

$$
\begin{aligned}
T_{i}: L^{2}\left(\partial \Omega_{i}\right) & \longrightarrow L^{2}\left(\partial \Omega_{i}\right) \\
v & \longmapsto T_{i} v \text { s.t. }\left(T_{i} v\right)_{i j}=R_{i j} v_{i j} \forall j .
\end{aligned}
$$

where $\left(T_{i} v\right)_{i i}=\left.\left(T_{i} v\right)\right|_{\partial \Omega_{i} \cap \Gamma}$ and $\left(T_{i} v\right)_{i j}=\left.\left(T_{i} v\right)\right|_{\partial \Omega_{i} \cap \partial \Omega_{j}}$. It follows that

$$
\begin{aligned}
J^{p+1} & =2 \sum_{i} \sum_{j} \int_{\partial \Omega_{i} \cap \partial \Omega_{j}}\left(T_{j}+T_{j}^{*}\right)^{-1}\left(\frac{\partial u_{j}^{p}}{\partial \mathbf{n}^{j}}+\mathbf{i} \omega T_{j}^{*} u_{j}^{p}\right)\left(\overline{\frac{\partial u_{j}^{p}}{\partial \mathbf{n}^{j}}+\mathbf{i} \omega T_{j}^{*} u_{j}^{p}}\right) \mathrm{d} \gamma \\
& =2 \sum_{j} \int_{\partial \Omega_{j}}\left(T_{j}+T_{j}^{*}\right)^{-1}\left(\frac{\partial u_{j}^{p}}{\partial \mathbf{n}^{j}}+\mathbf{i} \omega T_{j}^{*} u_{j}^{p}\right)\left(\overline{\frac{\partial u_{j}^{p}}{\partial \mathbf{n}^{j}}+\mathbf{i} \omega T_{j}^{*} u_{j}^{p}}\right) \mathrm{d} \gamma-\int_{\partial \Omega_{j} \cap \Gamma}\left|\frac{\partial u_{j}^{p}}{\partial \mathbf{n}^{j}}+\mathbf{i} \omega u_{j}^{p}\right|^{2} \mathrm{~d} \gamma
\end{aligned}
$$

Applying Lemma 6 to $\Omega_{j}$ and the boundary operator $T_{j}$ instead of $\Omega$ and $T$, it yields

$$
\begin{aligned}
J^{p+1} & =2 \sum_{j}\|\| \frac{\partial u_{j}^{p}}{\partial \mathbf{n}^{j}}+\mathbf{i} \omega T_{j}^{*} u_{j}^{p}\|\|^{2}-4 \omega^{2}\left\|u_{j}^{p}\right\|_{L^{2}\left(\partial \Omega_{j} \cap \Gamma\right)}^{2} \\
& =2 \sum_{j}\|\| \frac{\partial u_{j}^{p}}{\partial \mathbf{n}^{j}}-\mathbf{i} \omega T_{j} u_{j}^{p}\left\|\left.\right|^{2}-4 \omega^{2}\right\| u_{j}^{p} \|_{L^{2}\left(\partial \Omega_{j} \cap \Gamma\right)}^{2} \\
& =2 \sum_{j} \int_{\partial \Omega_{j}}\left(T_{j}+T_{j}^{*}\right)^{-1}\left(\frac{\partial u_{j}^{p}}{\partial \mathbf{n}^{j}}-\mathbf{i} \omega T_{j} u_{j}^{p}\right)\left(\frac{\partial u_{j}^{p}}{\partial \mathbf{n}^{j}}-\mathbf{i} \omega T_{j} u_{j}^{p}\right) \mathrm{d} \gamma-4 \omega^{2}\left\|u_{j}^{p}\right\|_{L^{2}\left(\partial \Omega_{j} \cap \Gamma\right)}^{2} \\
& =J^{p}+\sum_{j} \int_{\partial \Omega_{j} \cap \Gamma}\left|\frac{\partial u_{j}^{p}}{\partial \mathbf{n}^{j}}-\mathbf{i} \omega u_{j}^{p}\right|^{2} \mathrm{~d} \gamma-4 \omega^{2}\left\|u_{j}^{p}\right\|_{L^{2}\left(\partial \Omega_{j} \cap \Gamma\right)}^{2} \\
& =J^{p}-4 \omega^{2}\left\|u_{j}^{p}\right\|_{L^{2}\left(\partial \Omega_{j} \cap \Gamma\right)}^{2} \mathrm{~d} \gamma,
\end{aligned}
$$

and the proof is ended.

Remark 6 Combining the TC developed above and the ABC developed in section 3 should come down to adapting the operator $R_{i i}$ and the energy.

\section{Numerical experiments}

\subsection{Framework}

The corner conditions proposed in this paper can be integrated in any finite element framework without major difficulty. The numerical simulations presented in this section have been achieved using open-source software: GMSH ${ }^{1}$ [20] (mesh and post-processing), GetDP ${ }^{2}$ [12] (finite element solver) and its domain decomposition module GetDDM ${ }^{3}[30]$. The program written by the authors

\footnotetext{
1 http://gmsh.info/, commit number 418deb961, version 4.6.0, May 7th 2020

2 http://getdp.info/, version 3.3.0

3 http://onelab.info/GetDDM/
} 
is available online ${ }^{4}$ under open-source license. Every algorithm presented in Sections 3 and 4 are available. Readers are welcome to try and test the program as it has been designed to be user-friendly and easy to use on every major operating systems without a compiling process. Installation instructions are explained in the README.md file which is provided in the source code.

The implementation of the algorithms follows the same procedure as explained in [30]. The implementation of the DDM with TC (25) is detailed below, and is relatively similar for the DDMs with ABC from Section 3. For each segment $\Delta_{r}^{i j}$, auxiliary functions $\left(\varphi_{r}^{i j}\right)^{p}:=\left.R_{i j}\left(u_{i}^{p}\right)\right|_{\Delta_{r}^{i j}}$ and $\left(\psi_{r}^{i j}\right)^{p}:=\left.R_{j i}\left(u_{i}^{p}\right)\right|_{\Delta_{r}^{i j}}$ are introduced as well as the unknown $\left(g_{r}^{i j}\right)^{p}:=-\frac{\partial u_{j}^{p}}{\partial \mathbf{n}^{j}}-\left.R_{j i}\left(u_{i}^{p}\right)\right|_{\Delta_{r}^{i j}}=$ $-\frac{\partial u_{j}^{p}}{\partial \mathbf{n}^{j}}-\mathbf{i} \omega\left(\varphi_{r}^{i j}\right)^{p}$. The algorithm (25) is then implemented:

- Initialize $u_{i}^{0}$ for every subdomain.

- For $i=1, \ldots, N_{\mathrm{dom}}-1$, compute $u_{i}^{p+1}$

$$
\left\{\begin{aligned}
\left(-\Delta-\omega^{2}\right) u_{i}^{p+1} & =f, & & \text { in } \Omega_{i}, \\
\frac{\partial u_{i}^{p+1}}{\partial \mathbf{n}^{i}}-\mathbf{i} \omega\left(\varphi_{r}^{i j}\right)^{p+1} & =\left(g_{r}^{i j}\right)^{p}, & & \text { on } \Delta_{r}^{i j}, \forall j, \forall r \\
\left(\partial_{\mathbf{n}^{i}}-\mathbf{i} \omega\right) u_{i}^{p+1} & =0, & & \text { on } \partial \Omega_{i} \cap \Gamma .
\end{aligned}\right.
$$

- For $i, j=1, \ldots, N_{\text {dom }}-1, j \neq i$, for all $r$, compute the transmitted data on $\Delta_{r}^{i j}$

$$
\left(g_{r}^{j i}\right)^{p+1}=-\left.\frac{\partial u_{i}^{p+1}}{\partial \mathbf{n}^{i}}\right|_{\Delta_{r}^{i j}}-\mathbf{i} \omega\left(\psi_{r}^{i j}\right)^{p+1}=-\left(g_{r}^{i j}\right)^{p}-\mathbf{i} \omega\left(\left(\varphi_{r}^{i j}\right)^{p+1}+\left(\psi_{r}^{i j}\right)^{p+1}\right) .
$$

The whole algorithm is then recast into a linear system

$$
(I-A) g=b
$$

where $g$ is the vector containing every unknown $g_{r}^{i j}, b$ is the right-hand side and $A g$ is obtained by solving problems (26) and (27) with $f=0$. The linear system (28) can obviously be solved using a fast solver such as GMRES [29]. In the associated program, it is possible to chose between a Jacobi and a GMRES solver.

We point out that, first, contrary to [30], the two tangential transmission operators over an interface $\Delta^{i j}$ are not equal (i.e. $R_{i j} \neq R_{j i}$ ), and second, the goal of this section is to illustrate the stability of the different algorithms proposed in the paper, not to compare their efficiency with existing techniques.

\subsection{A simple test with analytical solution}

To validate the implementation of the second order transmission condition (3), we consider a simple wave guide problem. The domain is $\Omega:=(-D, D) \times(0, D)$, and the subdomains are $\Omega_{0}:=(-D, 0) \times(0, D)$ and $\Omega_{1}:=(0, D) \times(0, D)$, with a straight line interface $\Delta:=\{0\} \times(0, D)$. The equation in $\Omega$ is completed by the following boundary conditions. On the left boundary, we consider a non-homogeneous Robin condition $-\partial_{x} u(-D, y)-\mathbf{i} \omega u(-D, y)=g(y)$ for $0<y<D$. On the top and bottom boundaries, we take a homogeneous Neumann condition $\partial_{y} u(x, 0)=$ $\partial_{y} u(x, D)=0$ for $-D<x<D$. On the right boundary, we consider the simple absorbing boundary condition $\partial_{x} u(D, y)-\mathbf{i} \omega u(D, y)=0$ for $0<y<D$.

\footnotetext{
4 https://plmlab.math.cnrs.fr/ddm-corner-helmholtz-2d/scripts-onelab, commit 22263828, May 18th 2020
} 

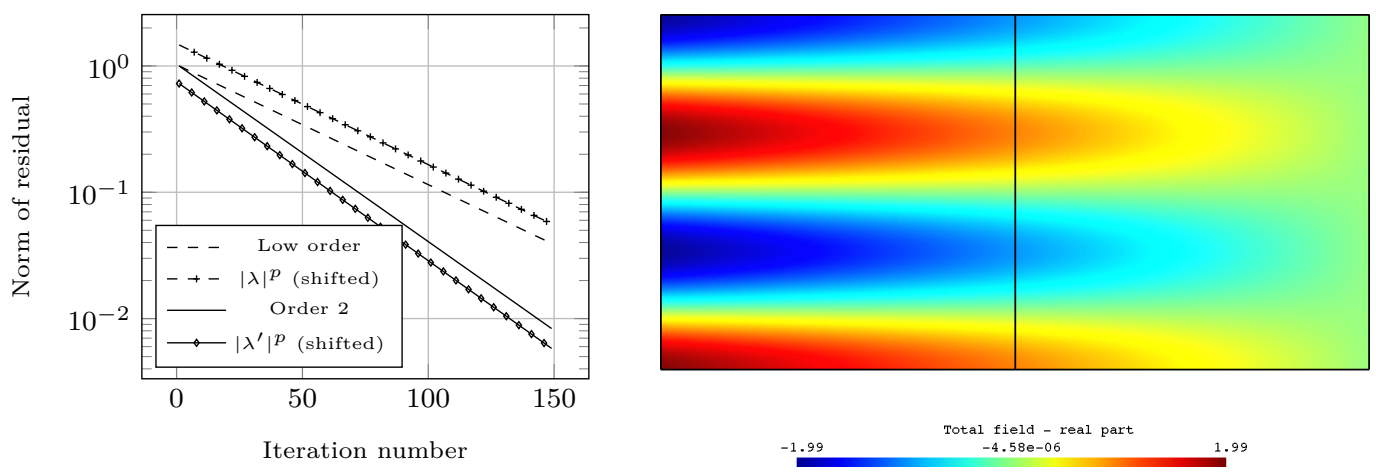

Fig. 6: Left: comparison of the residual's convergence for low order and $2^{\text {nd }}$ order TC, with references curves $|\lambda|^{p}$ and $\left|\lambda^{\prime}\right|^{p}$. Right: solution at (quasi-)convergence with DDM and $2^{\text {nd }}$ order TC.

The solution is computed with the DDM described in Section 4, where the transmission condition on the interface $\Delta$ is formulated either as a low order condition as in (18), or as a second order condition as in (23), where the operator $R_{i j}$ is the formal inverse of $L=\left(I-\frac{1}{2 \omega^{2}} \partial_{y y}\right)$. The operator $L$ is supplemented with homogeneous Neumann conditions at the two endpoints of the interface $\Delta$. To construct a simple analytical solution, we take a pure mode with homogeneous Neumann boundary data in the vertical direction

$$
g(y)=2 \cos (\omega y) \quad \text { with } \quad \omega=\frac{q \pi}{D} \quad \text { and } \quad q \in \mathbb{N} .
$$

With separation of variables in $\Omega_{1}$, a solution of $-\Delta u-\omega^{2} u=0$ with homogeneous Neumann $\partial_{y} u(x, 0)=\partial_{y} u(x, D)=0$ for $-D<x<D$ writes $u(x, y)=(\alpha+\beta x) \cos (\sigma y)$. The absorbing condition at $x=D$ writes $\beta-\mathbf{i} \omega(\alpha+\beta D)=0$, so that $(1-\mathbf{i} \omega D) \beta=\mathbf{i} \omega \alpha$.

For the low order DDM, define the transmission over $\Delta$ quantities

$$
\left\{\begin{array}{l}
a:=-\partial_{\mathbf{n}} u-\mathbf{i} \omega u=(\beta-\mathbf{i} \omega \alpha) \cos (\omega y)=\mathbf{i} \omega D \beta \cos (\omega y), \\
b:=\partial_{\mathbf{n}} u-\mathbf{i} \omega u=(-\beta-\mathbf{i} \omega \alpha) \cos (\omega y)=(-2+\mathbf{i} \omega D) \beta \cos (\omega y) .
\end{array}\right.
$$

The proportionality ratio between $a$ and $b$ is $\lambda=\frac{\mathbf{i} \omega D}{-2+\mathbf{i} \omega D}=\frac{\mathbf{i} q \pi}{-2+\mathbf{i} q \pi}$. Its modulus is also the modulus of a single iteration of DDM in $\Omega_{1}$, and is

$$
|\lambda|=\frac{|q| \pi}{\sqrt{4+q^{2} \pi^{2}}}<1 .
$$

A similar algebra can be performed in $\Omega_{0}$, but with a homogeneous Dirichlet boundary condition on the right boundary. Since this boundary condition is not absorbing, the modulus of the similar complex number $\lambda$ is now equal to 1 .

For the second order DDM, as $L g=\frac{3}{2} g$, the corresponding transmission over $\Delta$ quantities are now

$$
\left\{\begin{array}{l}
a^{\prime}:=-\partial_{\mathbf{n}} u-\frac{2}{3} \mathbf{i} \omega u=\left(\beta-\frac{2}{3} \mathbf{i} \omega \alpha\right) \cos \omega y=\left(\frac{1}{3}+\frac{2}{3} \mathbf{i} \omega D\right) \beta \cos \omega y, \\
b^{\prime}:=\quad \partial_{\mathbf{n}} u-\frac{2}{3} \mathbf{i} \omega u=\left(-\beta-\frac{2}{3} \mathbf{i} \omega \alpha\right) \cos \omega y=\left(-\frac{5}{3}+\frac{2}{3} \mathbf{i} \omega D\right) \beta \cos \omega y .
\end{array}\right.
$$

The modulus of the proportionality ratio $\lambda^{\prime}$ between $a^{\prime}$ and $b^{\prime}$ is now

$$
\left|\lambda^{\prime}\right|=\frac{\sqrt{1+4 q^{2} \pi^{2}}}{\sqrt{25+4 q^{2} \pi^{2}}}<1
$$


For the numerical illustration of Figure 6 , we chose $\omega=1, q=3$ and $D=3 \pi$. The values of the two modulus are $|\lambda| \approx 0.9782$ and $\left|\lambda^{\prime}\right| \approx 0.9679$. On the left of Figure 6 are plotted the residual's norms for the DDM with low and second order TC, against the reference curves $|\lambda|^{p}$ for low order and $\left|\lambda^{\prime}\right|^{p}$ for second order. The agreement is very good between the computed and the reference curves. On the right is plotted the quasi-converged solution computed with $2^{\text {nd }}$ order TC DDM. We consider that these results validate our implementation of the second order transmission condition.

\section{$5.3 \mathrm{ABC}$}

This second test case is devoted to the illustration of the new corner conditions (8). We consider a disk or radius $R=1$ inside an hexagon of diameter $L=10$, and compute the acoustic field diffracted by an incident plane wave $u_{\text {inc }}(x, y)=e^{\mathbf{i} \omega x}$. The diffracted field satisfies the homogeneous Helmholtz equation in the domain plus a non homogeneous Dirichlet boundary condition $u=-u_{\text {inc }}$ on the interior boundary. The exterior boundary is equipped with either: a) a low order $\mathrm{ABC}$; b) a second order $\mathrm{ABC}$ (3) with homogeneous Neumann conditions at the 6 corners of the exterior boundary; c) a second order ABC (3) with the corner condition (8) at the 6 corners of the exterior boundary. In what follows, the wavenumber $\omega$ is set to $2 \pi$, so that the wavelength is $\lambda=\frac{2 \pi}{\omega}=1$, and the mesh refinment is set to 15 points per wavelength. The direct solver MUMPS [1] is used to solve the volume (sub-)problems.

\subsubsection{Global $A B C$}

The numerical solution is computed using the global ABC, which we also call the "mono-domain" problem. In Figure 7, the first column corresponds to real part of the diffracted field with the three different ABCs. The second column corresponds to the norm of the difference with respect to the reference solution $u_{\text {ref }}$ in $\mathbb{R}^{2} \backslash\left\{x^{2}+y^{2} \leq R^{2}\right\}$, computed using a standard analytical method (Mie series). The results are qualitatively the same along the first column, with extra oscillations for the low order $\mathrm{ABC}$ (top right). The results from the second column show that the error may be due to the modeling at the rightmost corner. The magnitude of the error is of 0.245 for low order, 0.204 for second order ABC with Neumann and 0.152 with the new second order $\mathrm{ABC}(3)$ with corner conditions: it shows the ability of the new method to lower the corner errors.

\subsection{2 $D D M-1$}

Now that the different ABCs have been compared in the mono-domain problem, we compute the numerical solution for the $\mathrm{ABC}$ with the new corner condition using the DDM-1 algorithm (18) with 3 subdomains. The results are presented in Figure 8, using the Jacobi solver with 500 iterations (left column) and using the GMRES solver until convergence (67 iterations) for a tolerance of $10^{-6}$ (right column).

We first focus on the first column, and the Jacobi solver. The obtained solution (Figure 8a) is satisfactory and the error with respect to the mono-domain solution (Figure 8c) is located on the transmission boundaries. The algorithm is convergent (Figure 8e), as predicted by Lemma 7 and Remark 2. Convergence is, however, very slow, and oscillating. The histories of convergence for the two other ABCs (low order and $2^{\text {nd }}$ order with homogeneous Neumann) are also displayed, and we observe that the convergence rate is not really affected by the $\mathrm{ABC}$, which is quite natural. 


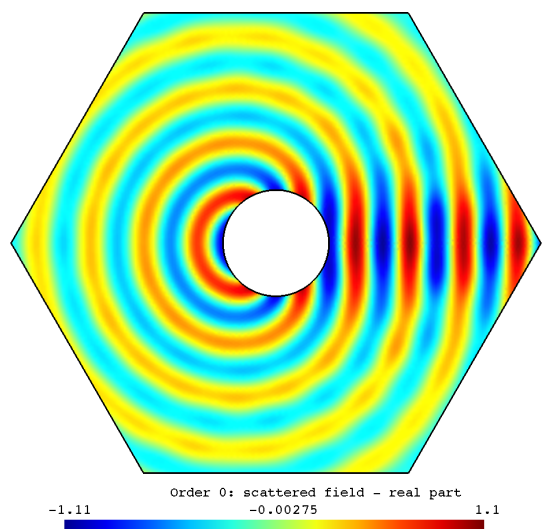

(a) $\operatorname{Re}(u)$ : Order 0

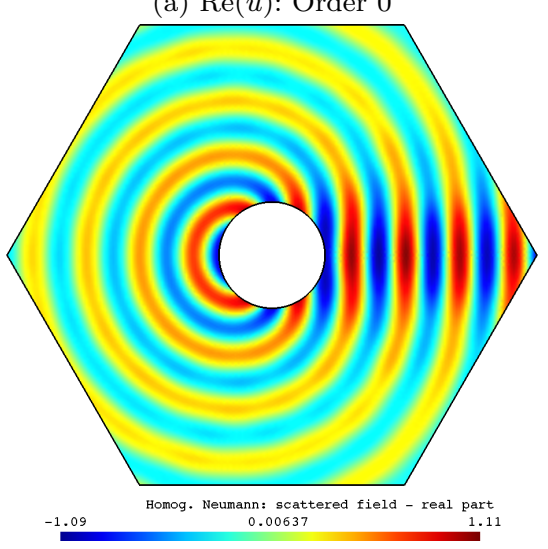

(c) $\operatorname{Re}(u)$ : Order $2+$ Homog. Neumann

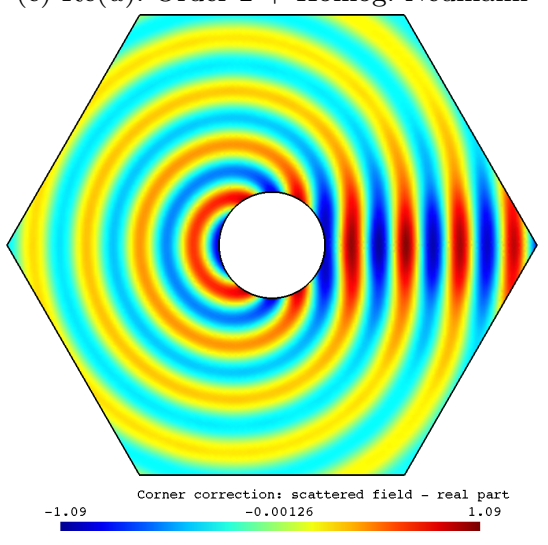

(e) $\operatorname{Re}(u)$ : Order $2+$ Corner correction

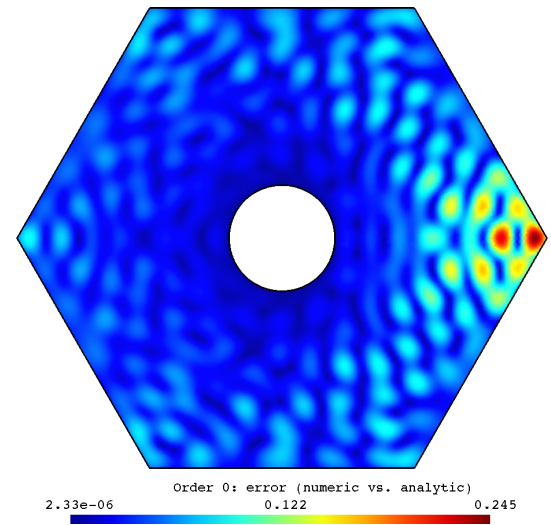

(b) $\left|u-u_{\text {ref }}\right|$ : Order 0

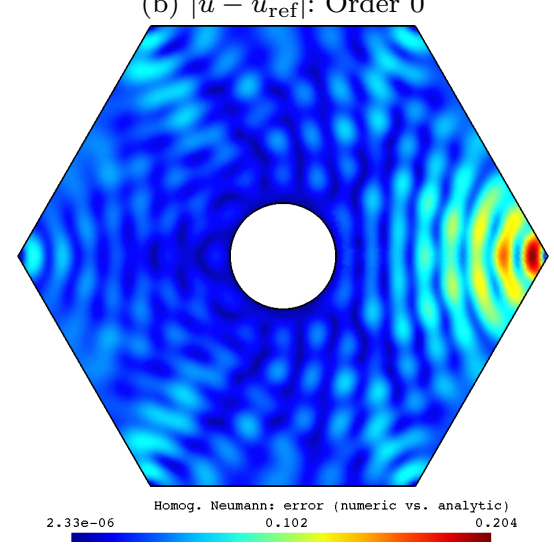

(d) $\left|u-u_{\text {ref }}\right|:$ Order $2+$ Homog. Neumann

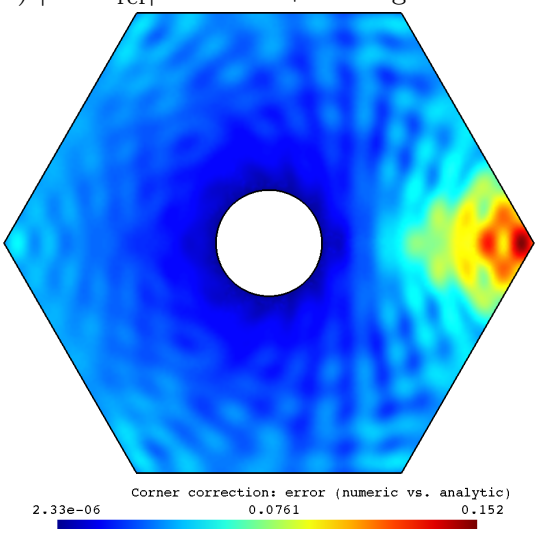

(f) $\left|u-u_{\text {ref }}\right|:$ Order $2+$ Corner correction

Fig. 7: Global problem solved using a direct solver (MUMPS) and the three different ABCs. Left: real part of the resulting scattered field. Right: error with respect to the reference solution. Parameters are: wavenumber $\omega=2 \pi$, unit disk sound-soft obstacle (Dirichlet condition) and hexagonal diameter of $L=10$. Incident plane wave $u_{\text {inc }}(x, y)=e^{\mathbf{i} \omega x}$. Mesh refinement set to 15 points per wavelength. 
On the right column, the same tests were done using the GMRES solver. The results lead to the same conclusion as for the Jacobi case: the solution is satisfactory (Figure 8b) and the ABC does not play a central role in the convergence rate. The magnitude of the error with respect to the mono-domain solution is now of the order of the tolerance rate (Figure 8d).

\subsubsection{Comparison DDM-2/DDM-1}

We now want to assess the convergence of algorithm DDM-2 (19)-(20) which is more local than DDM-1, since the ABC on the exterior boundary is split by using another decomposition method level. The results for DDM-2 after 1000 iterations of the Jacobi solver is shown in Figure 9a for the absolute value of the real part of the diffracted field. The reflection region is visible on the right of the obstacle. The error with respect to the DDM-1 solution (with 1000 iterations of Jacobi) is shown in Figure 9b. This error is removed when using a converged GMRES. The results confirm that the solution at convergence is the same for both DDM-2 and DDM-1 algorithms.

On the bottom line, Figures 9d-9c correspond to DDM-2 for the two other ABCs. These results are also the same as one would obtained with DDM-1.

Less oscillations are observed in Figure 9a, and we conclude that the results are better with the $2^{\text {nd }}$ order $\mathrm{ABC}$ and new corner conditions than without the corner condition or with low order ABC, for which algorithms DDM-2 and DDM-1 become identical.

\section{$5.4 \mathrm{TC}$}

We consider a transmission problem for a wave guide with the geometry depicted in Figure 5. The domain is a square $\Omega=(0,10)^{2}$ with two subdomains. The wavenumber is set to $\omega=\pi$ and the mesh refinement to 15 points per wavelength. The interface connects the corner and endpoints, from bottom to top: $\mathbf{P}=(5,-5), \mathbf{Q}_{0}=(5,-2), \mathbf{Q}_{1}=(6,0), \mathbf{Q}_{2}=(5,2)$ and $\mathbf{P}^{\prime}=(5,5)$. The boundary conditions are: non homogeneous Robin $-\left(\partial_{x}+\mathbf{i} \omega\right) u(0, y)=g$ on the left, homogeneous Robin $\partial_{x} u(10, y)-\mathbf{i} \omega u(10, y)=0$ on the right, and homogeneous Neumann on the top and bottom boundaries. The source is a plane wave $g=-\left(\partial_{x}+\mathbf{i} \omega\right) e^{\mathbf{i} \omega x}$, so that the analytical solution is known and is the same plane wave $u(x, y)=e^{\mathrm{i} \omega x}$. With this test, we can measure the numerical errors generated by the three corners $\mathbf{Q}_{0,1,2}$ in the regime of nearly normal incidence at the corners.

On the left of Figure 10 are plotted the norm of the residual computed with 4 different TCs. The first one is the classical low order TC and, without surprise, we recover a low rate of convergence. The three other ones are computed with different $2^{\text {nd }}$ order conditions, labeled Order 2 , where $R$ is constructed as the inverse of the second order tangential operator $R=\left(I-\frac{1}{2 \omega^{2}} \partial_{t t}\right)^{-1}$, with Neumann boundary conditions at $\mathbf{P}$ and $\mathbf{P}^{\prime}$, but with 3 different conditions at $\mathbf{Q}_{0,1,2}$ : continuity, labeled Dirichlet; homogeneous Neumann, labeled Neumann; the corner conditions from Section 4, labeled Corner Correction. In terms of the asymptotic rate of convergence, the new corner conditions are not a great improvement with respect to the three other conditions. The constant is however much better as the level of the plateau is lower, which is already an important improvement.

To evaluate the differences between the three $2^{\text {nd }}$ order TCs, Figure 11 shows the values of the function $R\left(u^{p}\right)$ along the interface at iteration $p=10$. For this test, another source $g(y)$ calculated from a pure plane wave which propagates at 45 degrees is used. It is interesting to notice that the value of $R\left(u^{p}\right)$ brings information about the corners values of the normal derivatives $\partial_{\mathbf{n}_{0,1}} u^{p}$. Indeed, starting from a zero initial iterate $u^{0}=0$, the TCs (25) propagate the homogeneous Neumann boundary conditions at $\mathbf{Q}_{0,1,2}$, see the center plot, while the normal derivatives of 


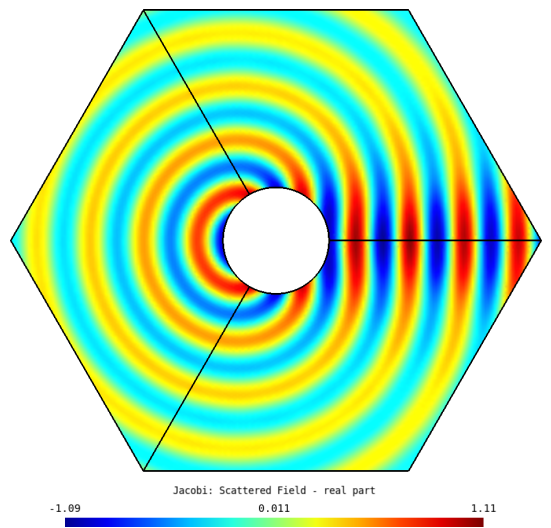

(a) Jacobi : $\operatorname{Re}(u)$

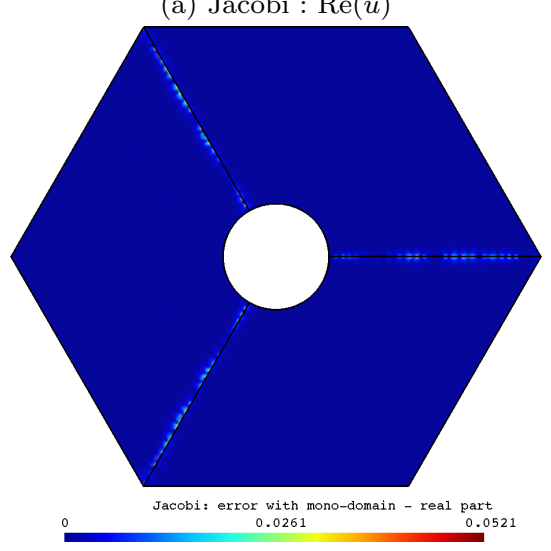

(c) Jacobi: $\left|u-u_{\text {mono }}\right|$

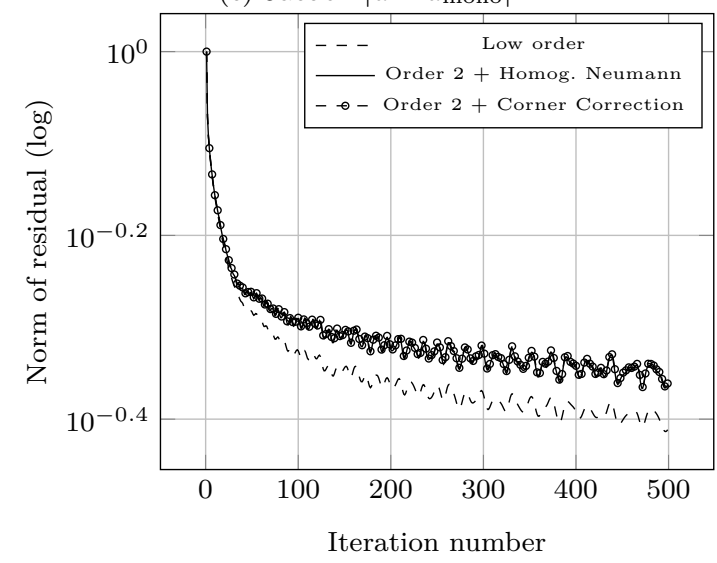

(e) Jacobi: History of convergence

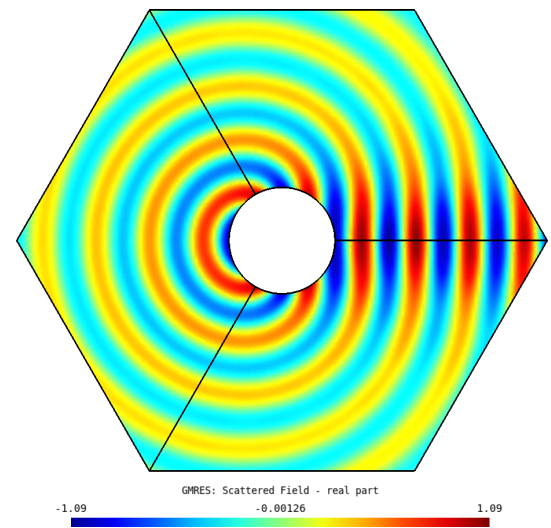

(b) GMRES : Re(u)

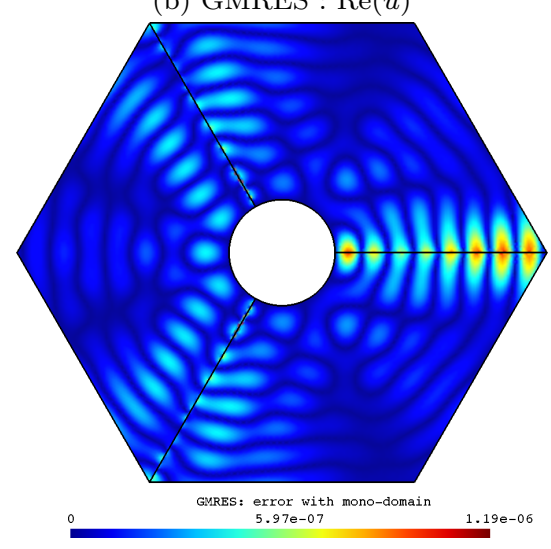

(d) GMRES: $\left|u-u_{\text {mono }}\right|$

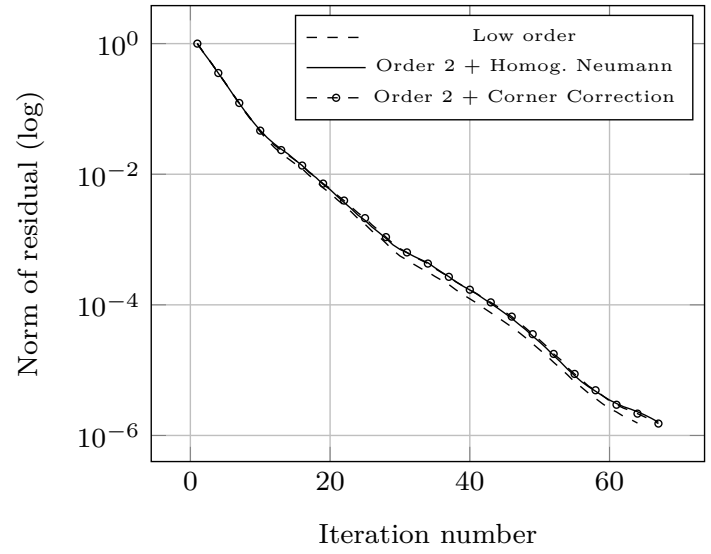

(f) GMRES: History of convergence

Fig. 8: Results obtained with DDM-1 and Jacobi solver (left) and GMRES solver (right). For each solver are displayed: the real part of the scattered field with the new ABC (top), the error with the mono-domain solution with the new ABC (center) and the history of convergence with the three ABCs (bottom). Parameters are the same as in Figure 7. 


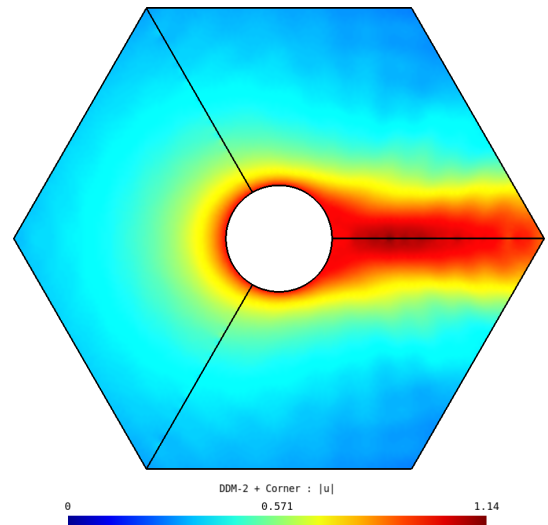

(a) Corner correction: $|u|$

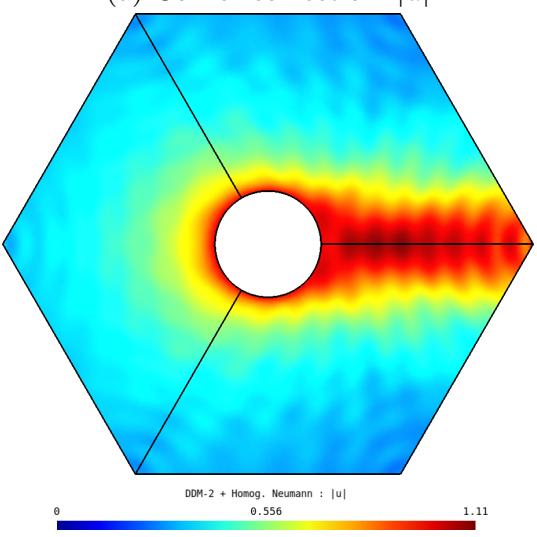

(c) Homog. Neumann: $|u|$

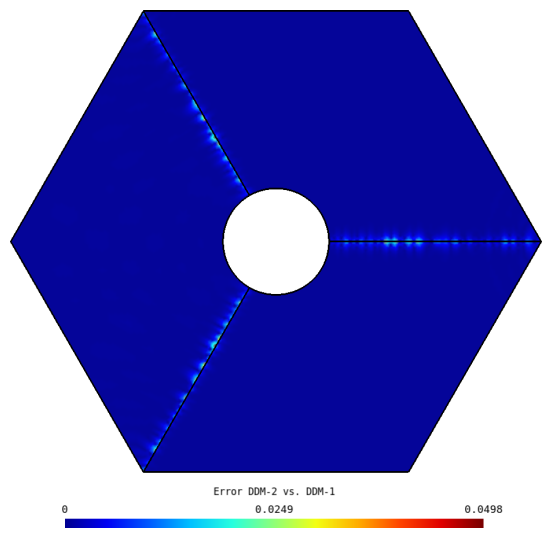

(b) Corner correction: $\left|u_{\mathrm{DDM}-1}-u_{\mathrm{DDM}-2}\right|$

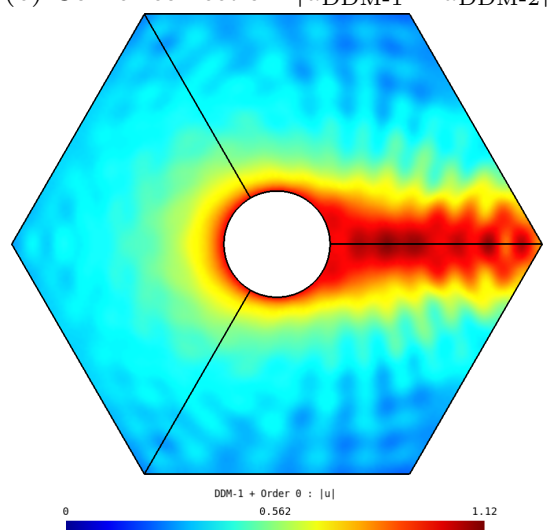

(d) Low order: $|u|$

Fig. 9: Absolute value of the real part of the diffracted field using DDM-2 after 1000 iterations with Jacobi solver. Second order ABC with (a) corner correction, (c) homogeneous Neumann. (d) Low order ABC. (b) Error between DDM-2 and DDM-1. Parameters are the same as in Figures 7 and 8.

the exact solution $\partial_{\mathbf{n}_{0,1}} u^{\text {exact }}$ do not satisfy such artificial conditions. The Dirichlet continuity relations, see the left plot, are also artificial. On the contrary, the new corner conditions do not propagate such simple and erroneous corners conditions, see the right plot. It is however an open problem to understand in more details the new corner conditions' nature.

\section{A Geometric interpretation of the $\mathrm{ABC}$}

To provide a geometric intuition of the different terms of the bilinear form $a$ from (14), we consider the simple situation where the domain is a $K$ sided regular polygon approximating the disc $\mathcal{D}=\left\{x^{2}+y^{2} \leq R^{2}\right\}$ from the interior as $K$ goes to infinity. The corners of the polygon are denoted $A_{k}=R\left(\cos \frac{2 k \pi}{K}, \sin \frac{2 k \pi}{K}\right)$ with $k$ defined modulo $K$. The middle of the edges are the $A_{k+1 / 2}=R\left(\cos \frac{(2 k+1) \pi}{K}, \sin \frac{(2 k+1) \pi}{K}\right)$. With our convention, see Figure 2, the interior angle is $\theta_{K}=\frac{2 \pi}{K}-\pi \in(-\pi, 0)$. The arclength between two successive corners is $\ell_{K}=\frac{2 \pi}{K} R$. 

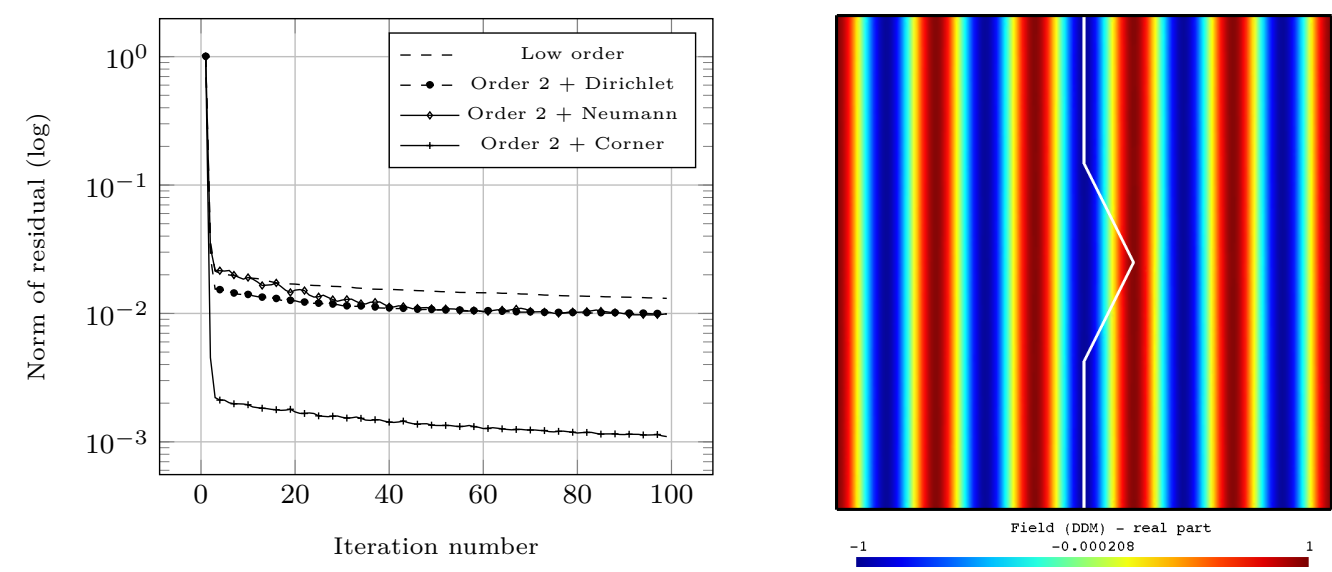

Fig. 10: Left: comparison between the convergence of the residuals for low order TC and $2^{\text {nd }}$ order TC with Dirichlet, homogeneous Neumann and the new corner conditions developed in this work. Right: solution at (quasi-)convergence for the DDM with new TC.
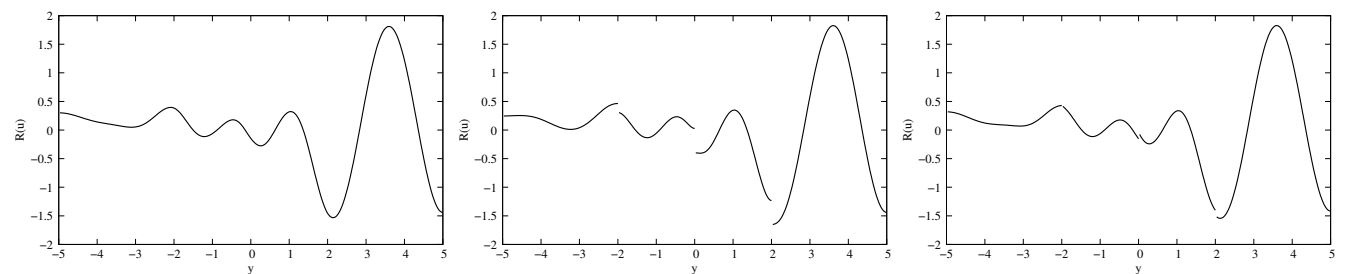

Fig. 11: Value of $R\left(u^{p}\right)$ with $p=10, R=\left(I-\frac{1}{2 \omega^{2}} \partial_{t t}\right)^{-1}$ and different conditions at corners $\mathbf{Q}_{0,1,2}$. Left: Dirichlet continuity relations. Center: homogeneous Neumann conditions. Right: the new corner conditions.

Let $\varphi$ and $\psi$ be two regular functions defined on the circle $\partial \mathcal{D}=\left\{x^{2}+y^{2}=R^{2}\right\}$. First, the Hermitian part of $a$, defined on the first line of (14), is a simple broken approximation of the integral

$$
\mathcal{I}_{1}(\varphi, \psi):=\int_{\partial \mathcal{D}}\left(\varphi(s) \overline{\psi(s)}+\frac{1}{2 \omega^{2}} \varphi^{\prime}(s) \overline{\psi^{\prime}(s)}\right) d s
$$

where $s$ is the curvilinear abscissa. Consider now the anti-Hermitian part of $a$, defined on the second line of (14). We study the two quantities

$$
\begin{aligned}
\mathcal{A}_{K} & :=\sum_{k} \cos \left(\frac{\theta_{K}}{2}\right)\left(\varphi\left(A_{k+1 / 2}\right)+\varphi\left(A_{k-1 / 2}\right)\right) \overline{\left(\psi\left(A_{k+1 / 2}\right)+\psi\left(A_{k-1 / 2}\right)\right)}, \\
\mathcal{B}_{K} & :=\sum_{k} \frac{\cos \left(\theta_{K}\right)}{\cos \left(\frac{\theta_{K}}{2}\right)}\left(\varphi\left(A_{k+1 / 2}\right)-\varphi\left(A_{k-1 / 2}\right)\right) \overline{\left(\psi\left(A_{k+1 / 2}\right)-\psi\left(A_{k-1 / 2}\right)\right)} .
\end{aligned}
$$

Lemma 11 As $K \rightarrow \infty$, one has $\mathcal{A}_{K} \rightarrow \mathcal{I}_{2}(\varphi, \psi):=\frac{2}{R} \int_{\partial \mathcal{D}} \varphi(s) \overline{\psi(s)} d s$.

Proof For large $K, \cos \left(\frac{\theta_{K}}{2}\right) \sim \frac{\ell_{K}}{2 R}$. Therefore $\mathcal{A}_{K}=\sum_{k} \frac{\ell_{K}}{2 R} 2 \varphi\left(A_{k}\right) \overline{2 \psi\left(A_{k}\right)}+$ high order terms. One recognizes a Riemann sum, and passing to the limit yields the claim.

Lemma 12 As $K \rightarrow \infty$, one has $\mathcal{B}_{K} \rightarrow \mathcal{I}_{3}(\varphi, \psi):=-2 R \int_{\partial \mathcal{D}} \varphi^{\prime}(s) \overline{\psi^{\prime}(s)} d s$. 
Proof For large $K, \frac{\cos \theta_{K}}{\cos \left(\frac{\theta_{K}}{2}\right)} \sim \frac{-2 R}{\ell_{K}}$. Therefore $\mathcal{B}_{K}=\sum_{k} \frac{-2 R}{\ell_{K}} \ell_{K} \varphi^{\prime}\left(A_{k}\right) \overline{\ell_{K} \psi^{\prime}\left(A_{k}\right)}+$ high order terms. Again, passing to the limit yields the claim.

The sesquilinear form $\varphi, \psi \mapsto a(\varphi, \psi)$ is thus an approximation of the sesquilinear form

$$
\widetilde{a}(\varphi, \psi):=\mathcal{I}_{1}(\varphi, \psi)-\frac{i}{4 \omega}\left(\mathcal{I}_{2}(\varphi, \psi)+\mathcal{I}_{3}(\varphi, \psi)\right),
$$

where the Hermitian part $\mathcal{I}_{1}$ is independent of the curvature radius $R$, and where the anti-Hermitian part $-\frac{i}{4 \omega}\left(\mathcal{I}_{2}+\right.$ $\mathcal{I}_{3}$ ) depends on the curvature radius via terms proportional to $R$ and $1 / R$. Similarily, the sesquilinear form $\varphi, \psi \mapsto a^{*}(\varphi, \psi)$ approximates the sesquilinear form $\widetilde{a}^{*}(\varphi, \psi):=\mathcal{I}_{1}(\varphi, \psi)+\frac{i}{4 \omega}\left(\mathcal{I}_{2}(\varphi, \psi)+\mathcal{I}_{3}(\varphi, \psi)\right)$. In classical planar differential geometry, a smooth curve $\Gamma$ locally separates the domain into two regions $\Omega_{ \pm}$. The curvature radius $R$ of $\Gamma$ seen from $\Omega_{+}$is the opposite of the curvature radius $-R$ of $\Gamma$ seen from $\Omega_{-}$. Therefore, another possible interpretation of the difference between $\widetilde{a}$ and $\widetilde{a}^{*}$ is that they correspond to the same curve $\Gamma$ seen from one side or the other.

Remark 7 Considering Remark 1, the same interpretation holds for $T$ and $T^{*}$ : they account for the curvature radius, and a change of sign of the curvature radius changes $T$ in $T^{*}$ and reciprocally.

The ABC associated to $\widetilde{a}(\varphi, \psi)=(u, \psi)_{L^{2}(\Gamma)}$, noticing that $\varphi \simeq(\mathbf{i} \omega)^{-1} \partial_{\mathbf{n}} u$, writes in its strong form

$$
\left(1-\frac{1}{2 \omega^{2}} \partial_{s s}-\frac{\mathbf{i}}{4 \omega}\left(\frac{2}{R}+2 R \partial_{s s}\right)\right) \partial_{\mathbf{n}} u-\mathbf{i} \omega u=0 .
$$

In polar coordinates and on the border of the disk $\mathcal{D}$, the normal derivative is the derivative along $r$ ( $\varphi \simeq$ $\left.(\mathbf{i} \omega)^{-1} \partial_{r} u\right)$ and the curvilinear derivative is given by $\partial_{s s}=\frac{1}{R^{2}} \partial_{\theta \theta}$. Hence the following strong form of the ABC in polar coordinates:

$$
\left(1-\frac{1}{2 R^{2} \omega^{2}} \partial_{\theta \theta}-\frac{\mathbf{i}}{2 \omega R}\left(1+\partial_{\theta \theta}\right)\right) \partial_{r} u-\mathbf{i} \omega u=0 .
$$

When the radius of the disk $R$ goes to infinity, the border of $\mathcal{D}$ tends to be (locally) straight and the ABC converges towards the classical low order $\mathrm{ABC} \partial_{r} u-\mathbf{i} \omega u=0$.

\section{B Subdomains and unknowns decoupled: DDM-3}

In this appendix, we modify algorithm DDM-2 to decouple (19) from (20), at the price of introducing a new auxiliary unknown on each edge $\Gamma_{k}^{i}$. This unknown, denoted $\psi_{i, k}$, represents the Dirichlet trace of $u_{i}$ on $\Gamma_{k}^{i}$. The interest is that $u_{i}^{p+1}$ can be obtained by solving a classical Helmholtz boundary value problem, where the boundary condition involves $\left(\varphi_{i, k}^{p}\right)_{k}$ and $\left(\psi_{i, k}^{p}\right)_{k}$ at the previous iteration index $p$.

Initialize $u_{i}^{0} \in H^{1}\left(\Omega_{i}\right)$ with square integrable normal derivatives on each subdomain, and $\left(\varphi_{i, k}^{0}\right)_{k} \in \oplus_{k} H^{1}\left(\Gamma_{k}^{i}\right)$, $\left(\psi_{i, k}^{0}\right)_{k} \in \oplus_{k} L^{2}\left(\Gamma_{k}^{i}\right)$ on the exterior boundary of each subdomain. For $p=0,1, \ldots$, solve for each subdomain

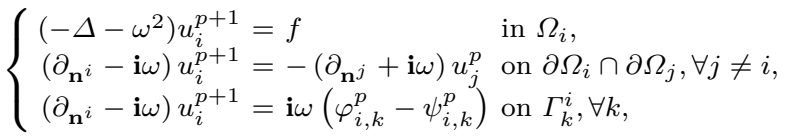

and for each edge

$$
\begin{cases}\left(1-\frac{1}{2 \omega^{2}} \partial_{\mathbf{t}_{k}} \mathbf{t}_{k}\right) \varphi_{i, k}^{p+1}(\mathbf{x})=\psi_{i, k}^{p+1}(\mathbf{x}), & \mathbf{x} \in \Gamma_{k}^{i}, \\ \varphi_{i, k}^{p+1}(\mathbf{x})+\psi_{i, k}^{p+1}(\mathbf{x})=\frac{1}{\mathbf{i} \omega} \frac{\partial u_{i}^{p}}{\partial \mathbf{n}^{i}}(\mathbf{x})+u_{i}^{p}(\mathbf{x}), & \mathbf{x} \in \Gamma_{k}^{i}, \\ \left(\left(1+\frac{\mathbf{i} \beta}{\omega} \frac{\cos \left(\frac{\theta_{k \ell}}{2}\right)}{\cos \theta_{k \ell}}\right) \frac{\partial \varphi_{i, k}^{p+1}}{\partial \tau_{k}}+\left(\beta-\mathbf{i} \omega \cos \left(\frac{\theta_{k \ell}}{2}\right)\right) \varphi_{i, k}^{p+1}\right)\left(\mathbf{A}_{k \ell}^{i j}\right) & \\ =\left(\left(-1+\frac{\mathbf{i} \beta}{\omega} \frac{\cos \left(\frac{\theta_{k \ell}}{2}\right)}{\cos \theta_{k \ell}}\right) \frac{\partial \varphi_{j, \ell}^{p}}{\partial \boldsymbol{\tau}_{\ell}}+\left(\beta+\mathbf{i} \omega \cos \left(\frac{\theta_{k \ell}}{2}\right)\right) \varphi_{j, \ell}^{p}\right)\left(\mathbf{A}_{k \ell}^{i j}\right), \mathbf{A}_{k \ell}^{i j} \in \mathcal{C}_{k}^{i}, \\ \left(\mathbf{i} \omega \varphi_{i, k}^{p+1}+\partial_{\boldsymbol{\tau}_{k}} \varphi_{i, k}^{p+1}\right)\left(\mathbf{B}_{k}^{i j}\right)=\left(\mathbf{i} \omega \varphi_{j, k}^{p}-\partial_{\boldsymbol{\tau}_{k}} \varphi_{j, k}^{p}\right)\left(\mathbf{B}_{k}^{i j}\right), & \mathbf{B}_{k}^{i j} \in \mathcal{F}_{k}^{i},\end{cases}
$$


with the same $\beta$ as before to remove the singularity, see Remark 3. We now show the algorithm is endowed with a decreasing energy for $f=0$. Define

$$
\begin{aligned}
G^{p}:= & \sum_{i=0}^{N_{\mathrm{dom}}-1}\left(\int_{\partial \Omega_{i} \backslash \Gamma}\left|\left(\partial_{\mathbf{n}^{i}}-\mathbf{i} \omega\right) u_{i}^{p}\right|^{2} \mathrm{~d} \gamma+\sum_{k=0}^{K-1} \int_{\Gamma_{k}^{i}} \omega^{2}\left|\varphi_{i, k}^{p}+\psi_{i, k}^{p}\right|^{2} \mathrm{~d} \gamma\right. \\
& +\sum_{\substack{k=0 \\
\mathbf{A}_{k \ell}^{i j} \in \mathcal{C}_{k}^{i}}}^{K-1} \frac{1}{2|\omega| \sin ^{2}\left(\frac{\theta_{k \ell}}{2}\right)}\left|\left(1+\frac{\mathbf{i} \beta}{\omega} \frac{\cos \left(\frac{\theta_{k \ell}}{2}\right)}{\cos \theta_{k \ell}}\right) \frac{\partial \varphi_{i, k}^{p}}{\partial \tau_{k}}+\left(\beta-\mathbf{i} \omega \cos \left(\frac{\theta_{k \ell}}{2}\right)\right) \varphi_{i, k}^{p}\right|^{2}\left(\mathbf{A}_{k \ell}^{i j}\right) \\
& \left.+\sum_{\substack{k=0 \\
\mathbf{B}_{k}^{i j} \in \mathcal{F}_{k}^{i}}}^{K-1} \frac{1}{2 \omega}\left|\omega \varphi_{i, k}^{p}+\partial_{\boldsymbol{\tau}_{k}} \varphi_{i, k}^{p}\right|^{2}\left(\mathbf{B}_{k}^{i j}\right)\right)=F^{p}+\sum_{i=0}^{N_{\mathrm{dom}}-1 K-1} \sum_{k=0} \int_{\Gamma_{k}^{i}} \omega^{2}\left|\varphi_{i, k}^{p}+\psi_{i, k}^{p}\right|^{2} \mathrm{~d} \gamma
\end{aligned}
$$

Lemma 13 The algorithm (29)-(30) is stable. For $f=0$, it has decreasing energy

$$
G^{p+1}=G^{p}-2 \sum_{j=0}^{N_{d o m}-1} \sum_{\ell=0}^{K-1} \int_{\Gamma_{\ell}^{j}}\left(2 \omega^{2}\left|\varphi_{j, \ell}^{p}\right|^{2}+\left|\partial_{\mathbf{t}_{\ell}} \varphi_{j, \ell}^{p}\right|^{2}\right) \mathrm{d} \gamma
$$

Proof Similar computations to the ones of the proof of Lemma 8 give

$$
G^{p+1}=G^{p}-4 \operatorname{Re} \sum_{j=0}^{N_{\mathrm{dom}}} \sum_{\ell=0}^{-1} \int_{\Gamma_{\ell}^{j}} \omega^{2} \varphi_{j, \ell}^{p} \overline{\psi_{j, \ell}^{p}} \mathrm{~d} \gamma-2 \operatorname{Re} \sum_{j=0}^{N_{\mathrm{dom}}-1} \sum_{\substack{\ell=0 \\ \mathbf{A} \in \mathcal{C}_{\ell}^{j} \cup \mathcal{F}_{\ell}^{j}}}^{K-1} \frac{\partial \varphi_{j, \ell}^{p}}{\partial \tau_{\ell}}(\mathbf{A}) \overline{\varphi_{j, \ell}^{p}}(\mathbf{A}) .
$$

Integrating the first equation of system (30) at iteration $p$ on $\Gamma_{\ell}^{j}$ against $\varphi_{j, \ell}^{p}$ and taking the sum over all subdomain and edge indices $j$ and $\ell$ gives the result:

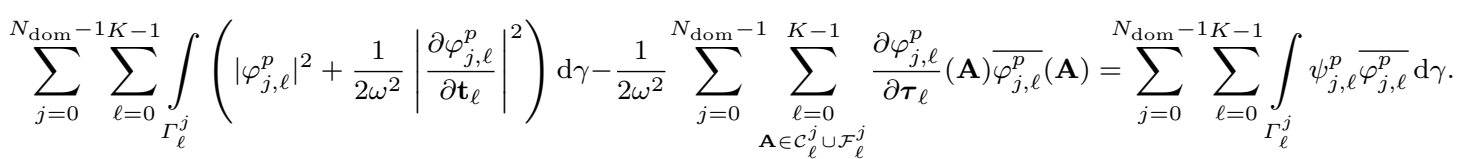

\section{References}

1. P. Amestoy, I. S. Duff, J. Koster, and J.-Y. L'Excellent. A fully asynchronous multifrontal solver using distributed dynamic scheduling. SIAM Journal on Matrix Analysis and Applications, 23(1):15-41, 2001.

2. A. Bamberger, P. Joly, and J. E. Roberts. Second-order absorbing boundary conditions for the wave equation: A solution for the corner problem. SIAM Journal on Numerical Analysis, 27(2):323-352, 1990.

3. J.-D. Benamou and B. Desprès. A domain decomposition method for the helmholtz equation and related optimal control problems. Journal of Computational Physics, 136(1):68 - 82, 1997.

4. A. Bendali and Y. Boubendir. Non-overlapping domain decomposition method for a nodal finite element method. Numer. Math., 103(4):515-537, 2006.

5. Y. Boubendir, X. Antoine, and C. Geuzaine. A quasi-optimal non-overlapping domain decomposition algorithm for the Helmholtz equation. J. Comput. Phys., 231(2):262-280, 2012.

6. Y. Boubendir, A. Bendali, and M. B. Fares. Coupling of a non-overlapping domain decomposition method for a nodal finite element method with a boundary element method. Internat. J. Numer. Methods Engrg., 73(11):1624-1650, 2008.

7. C. Chniti, F. Nataf, and F. Nier. Improved interface conditions for $2 \mathrm{D}$ domain decomposition with corners: numerical applications. J. Sci. Comput., 38(2):207-228, 2009.

8. X. Claeys. A new variant of the optimised schwarz method for arbitrary non-overlapping subdomain partitions, 2019.

9. B. Després. Domain decomposition method and the Helmholtz problem. In Mathematical and numerical aspects of wave propagation phenomena (Strasbourg, 1991), pages 44-52. SIAM, Philadelphia, PA, 1991. 
10. C. R. Dohrmann, A. Klawonn, and O. B. Widlund. Extending theory for domain decomposition algorithms to irregular subdomains. In Domain decomposition methods in science and engineering XVII, volume 60 of Lect. Notes Comput. Sci. Eng., pages 255-261. Springer, Berlin, 2008.

11. V. Dolean, P. Jolivet, and F. Nataf. An Introduction to Domain Decomposition Methods. Society for Industrial and Applied Mathematics, Philadelphia, PA, 2015.

12. P. Dular and C. Geuzaine. GetDP reference manual: the documentation for GetDP, a general environment for the treatment of discrete problems. http://getdp.info.

13. B. Engquist and A. Majda. Absorbing boundary conditions for the numerical simulation of waves. Math. Comp., 31(139):629-651, 1977.

14. C. Farhat, P. Avery, R. Tezaur, and J. Li. FETI-DPH: a dual-primal domain decomposition method for acoustic scattering. J. Comput. Acoust., 13(3):499-524, 2005.

15. M. J. Gander. Optimized Schwarz methods. SIAM J. Numer. Anal., 44(2):699-731, 2006.

16. M. J. Gander and F. Kwok. Best Robin parameters for optimized Schwarz methods at cross points. SIAM J. Sci. Comput., 34(4):A1849-A1879, 2012.

17. M. J. Gander and F. Kwok. On the applicability of Lions' energy estimates in the analysis of discrete optimized Schwarz methods with cross points. In Domain decomposition methods in science and engineering XX, volume 91 of Lect. Notes Comput. Sci. Eng., pages 475-483. Springer, Heidelberg, 2013.

18. M. J. Gander, F. Magoulès, and F. Nataf. Optimized Schwarz methods without overlap for the Helmholtz equation. SIAM J. Sci. Comput., 24(1):38-60, 2002.

19. M. J. Gander and K. Santugini. Cross-points in domain decomposition methods with a finite element discretization. Electron. Trans. Numer. Anal., 45:219-240, 2016.

20. C. Geuzaine and J.-F. Remacle. Gmsh: A 3-D finite element mesh generator with built-in pre- and postprocessing facilities. Internat. J. Numer. Methods Engrg., 79(11):1309-1331, 2009. http://gmsh.info.

21. D. Gordon, R. Gordon, and E. Turkel. Compact high order schemes with gradient-direction derivatives for absorbing boundary conditions. J. Comput. Phys., 297:295-315, 2015.

22. L. Halpern and O. Lafitte. Dirichlet to Neumann map for domains with corners and approximate boundary conditions. J. Comput. Appl. Math., 204(2):505-514, 2007.

23. P. Joly, S. Lohrengel, and O. Vacus. Un résultat d'existence et d'unicité pour l'équation de Helmholtz avec conditions aux limites absorbantes d'ordre 2. C. R. Acad. Sci. Paris Sér. I Math., 329(3):193-198, 1999.

24. R. Kechroud, X. Antoine, and A. Soulaïmani. Numerical accuracy of a Padé-type non-reflecting boundary condition for the finite element solution of acoustic scattering problems at high-frequency. Internat. J. Numer. Methods Engrg., 64(10):1275-1302, 2005.

25. M. Lecouvez, B. Stupfel, P. Joly, and F. Collino. Quasi-local transmission conditions for non-overlapping domain decomposition methods for the helmholtz equation. Comptes Rendus Physique, 15(5):403 - 414, 2014. Electromagnetism / Électromagnétisme.

26. S. Loisel. Condition number estimates for the nonoverlapping optimized Schwarz method and the 2-Lagrange multiplier method for general domains and cross points. SIAM J. Numer. Anal., 51(6):3062-3083, 2013.

27. A. Modave, X. Antoine, and C. Geuzaine. An efficient domain decomposition method with cross-point treatment for Helmholtz problems. In CSMA 2019 - 14e Colloque National en Calcul des Structures, Giens (Var), France, May 2019.

28. A. Modave, C. Geuzaine, and X. Antoine. Corner treatments for high-order local absorbing boundary conditions in high-frequency acoustic scattering. Journal of Computational Physics, 401:109029, 2020.

29. Y. Saad and M. H. Schultz. GMRES: a generalized minimal residual algorithm for solving nonsymmetric linear systems. SIAM J. Sci. Statist. Comput., 7(3):856-869, 1986.

30. B. Thierry, A. Vion, S. Tournier, M. El Bouajaji, D. Colignon, N. Marsic, X. Antoine, and C. Geuzaine. Getddm: An open framework for testing optimized schwarz methods for time-harmonic wave problems. Computer Physics Communications, 203:309 - 330, 2016. 